\title{
The MCXC: a meta-catalogue of x-ray detected clusters of galaxies ${ }^{\star}$
}

\author{
R. Piffaretti ${ }^{1}$, M. Arnaud ${ }^{1}$, G. W. Pratt $^{1}$, E. Pointecouteau ${ }^{2}$, and J.-B. Melin ${ }^{3}$ \\ ${ }^{1}$ Laboratoire AIM, IRFU/Service d'Astrophysique - CEA/DSM - CNRS - Université Paris Diderot, Bât. 709, CEA-Saclay, \\ 91191 Gif-sur-Yvette Cedex, France \\ e-mail: rocco.piffaretti@cea.fr \\ 2 Université de Toulouse, CNRS, CESR, 9 av. du colonel Roche, BP 44346, 31028 Toulouse Cedex 04, France \\ 3 DSM/Irfu/SPP, CEA/Saclay, 91191 Gif-sur-Yvette Cedex, France
}

Received 12 July 2010 / Accepted 4 May 2011

\section{ABSTRACT}

\begin{abstract}
We present the compilation and properties of a meta-catalogue of X-ray detected clusters of galaxies, the MCXC. This very large catalogue is based on publicly available ROSAT All Sky Survey-based (NORAS, REFLEX, BCS, SGP, NEP, MACS, and CIZA) and serendipitous (160SD, 400SD, SHARC, WARPS, and EMSS) cluster catalogues. Data have been systematically homogenised to an overdensity of 500, and duplicate entries from overlaps between the survey areas of the individual input catalogues are carefully handled. The MCXC comprises 1743 clusters with virtually no duplicate entries. For each cluster the MCXC provides three identifiers, a redshift, coordinates, membership in the original catalogue, and standardised $0.1-2.4 \mathrm{keV}$ band luminosity $L_{500}$, total mass $M_{500}$, and radius $R_{500}$. The meta-catalogue additionally furnishes information on overlaps between the input catalogues and the luminosity ratios when measurements from different surveys are available, and gives notes on individual objects. The MCXC is available in electronic format for maximum usefulness in X-ray, SZ, and other multiwavelength studies.
\end{abstract}

Key words. X-rays: galaxies: clusters - cosmology: observations - large-scale structure of Universe - galaxies: clusters: general catalogs

\section{Introduction}

Clusters of galaxies provide cosmological constraints through the number density and evolution of objects, through the power spectrum of their three-dimensional distribution, and through their baryon fraction and its evolution. Moreover, the physical properties of clusters provide a test of the structure formation scenario, giving vital information for understanding the gravitational collapse of the dark matter and of the evolution of baryons in the dark matter potential (see Voit 2005, for a review).

$\mathrm{X}$-ray observations are ideal for these studies since the density squared dependence of the X-ray emission implies that clusters can be found efficiently over a wide redshift range. Cluster sources were evident in the first all-sky X-ray survey with Uhuru, and more objects were found by HEAO-1 and Ariel-V; subsequent follow-up observations with Einstein and EXOSAT allowed more accurate characterisation of their physical properties (see Rosati et al. 2002, for a review).

In this context, the ROSAT satellite has played a central role. The 1990-1991 ROSAT All-Sky Survey (RASS, Voges et al. 1999) and later deep pointed observations have led to the discovery of hundreds of clusters. Subsequent followup observations, in particular those conducted with the current generation of X-ray satellites XMM-Newton, Chandra, and Suzaku, have provided statistical samples for cosmological studies (e.g., Vikhlinin et al. 2009; Mantz et al. 2010b) and detailed information on the structural properties of the cluster population (e.g., Vikhlinin et al. 2006; Pratt et al. 2010; Arnaud et al. 2010).

\footnotetext{
* The catalog is only available at the CDS via anonymous ftp to cdsarc.u-strasbg.fr (130.79.128.5) or via

http://cdsarc.u-strasbg.fr/viz-bin/qcat?J/A+A/534/A109
}

Other X-ray observations have allowed in-depth study of the hierarchical assembly process through merging (e.g., Markevitch \& Vikhlinin 2007, and references therein) and the physical mechanisms associated with feedback and its impact on structure formation (e.g., McNamara \& Nulsen 2007, and references therein). However, while several XMM-Newton and Chandra X-ray surveys are ongoing (e.g., Romer et al. 2001; Barkhouse et al. 2006; Pacaud et al. 2007; Fassbender 2007) ${ }^{1}$, the associated cluster catalogues are either not yet published or only partially available.

Outside of the X-ray domain, the redshift-independent thermal Sunyaev-Zel'dovich effect (Sunyaev \& Zel'dovich 1972, hereafter SZ) is emerging as an efficient way to detect distant, massive clusters that fall below the flux limits of X-ray surveys. Several SZ surveys, including those being undertaken with the South Pole Telescope (SPT, Carlstrom et al. 2011) survey, the Atacama Cosmology Telescope (ACT, Fowler et al. 2007), and Planck (Tauber et al. 2010), are actively ongoing and have started to provide the first SZ-selected cluster samples (e.g., Vanderlinde et al. 2010; Menanteau et al. 2010; Planck Collaboration 2011d). X-ray observations of SZ-detected clusters are important in many respects. The X-ray properties allow a better characterisation of the SZ signal (e.g., Melin et al. 2006; Andersson et al. 2011; Planck Collaboration 2011g) and yield the calibration of the scaling relations needed for cosmological studies with SZ-selected cluster samples (e.g., Majumdar \& Mohr 2003). In addition, X-ray observations allow testing of the selection function of SZ surveys and verification of

\footnotetext{
${ }^{1}$ See http://cxc.harvard.edu/xraysurveys/surveys.html for a complete list of ongoing XMM-Newton and Chandra surveys.
} 
new SZ cluster candidates (e.g., Šuhada et al. 2010; Planck Collaboration 2011e). Moreover, they are essential for statistical analyses of the SZ data (e.g., Melin et al. 2011; Komatsu et al. 2010; Planck Collaboration 2011f,g,h, and references therein).

Cosmological tests that rely on knowledge of the evolution of the mass function or baryon fraction require an estimate of the cluster mass. Surveys provide only an observable (typically luminosity, temperature or SZ $y$-parameter) that is then linked to the cluster mass via scaling relations. While simultaneous constraints on cosmological parameters and scaling relations have recently been derived (Mantz et al. 2010a), the mass proxy relations are typically separately calibrated using deep observations of well-understood and if possible representative samples (e.g., Arnaud et al. 2007; Maughan 2007; Vikhlinin et al. 2009; Pratt et al. 2009). Although their redshift evolution is at present poorly known, a consensus on the type of scaling relations to be calibrated and their precise definition has been reached. For example, the bias introduced by cool core clusters in luminosity and temperature measurements is taken into account, low scatter mass proxies such as $Y_{\mathrm{X}}$ (Kravtsov et al. 2006) or the gas mass $M_{\text {gas }}$ are widely used, and all quantities are measured up to a standard characteristic radius $R_{500}$, the radius within which the mean over-density of the cluster is 500 times the critical density at the cluster redshift. Substantial progress has also been made in understanding the systematics affecting X-ray mass estimates via simulations (e.g., Rasia et al. 2006; Nagai et al. 2007a; Piffaretti \& Valdarnini 2008) and via combination with gravitational lensing (e.g., Mahdavi et al. 2008; Zhang et al. 2010; Meneghetti et al. 2010).

ROSAT-derived catalogues still play a major role in providing targets for deeper observation with the current generation of X-ray instruments (e.g., Böhringer et al. 2007; Vikhlinin et al. 2009), and for identification of existing clusters in new surveys in other wavelength bands (e.g., Popesso et al. 2004). These catalogues have been derived from a number of surveys based on RASS data or ROSAT pointed observations (see Sect. 2). Even though these catalogues are public, their large sizes and the different conventions employed (e.g., cosmology, integration radius etc.) mean that no attempt has yet been made to merge them and to homogenise the data contained within. The time is thus ripe for a major effort to standardise the information available in these catalogues.

Our aim in this paper is to provide such a service to the community by collecting the disparate measurements available in the major publicly-available X-ray cluster catalogues and regularising them to the same cosmology and integration radius. In particular, our approach is based on the use of the [0.1-2.4] keV X-ray luminosity as a mass proxy. Used together with a luminosity profile and the X-ray luminosity-mass scaling laws recently derived from the representative sample of X-ray clusters REXCESS, we standardise all measurements to an integration radius of $R_{500}$. The latter is chosen since it encloses a substantial fraction of the total virialised mass of the system, while being the largest radius probed in current high resolution X-ray observations of moderately large samples. Furthermore, use of the REXCESS luminosity profile and scaling laws ensures the best possible description of the average properties of the X-ray cluster population, and allows maximum self-consistency in our approach.

The basic characteristics of the resulting meta-catalogue of $\mathrm{X}$-ray detected clusters of galaxies (hereafter MCXC) are its large number of objects (1743 unique systems), its homogeneously estimated [0.1-2.4] keV band luminosities $\left(L_{500}\right)$ and total masses $\left(M_{500}\right)$, its use of a uniform format for all provided quantities, and its careful control of duplicate entries originating from overlaps between the input catalogues. In order to be easily manipulated, the MCXC is provided in electronic format. The final catalogue gives a first overview of the published, publiclyavailable X-ray survey selected cluster population.

Here it is worth clarifying what we envisage the MCXC is useful for. One purpose as a large, homogeneous catalogue is for cross correlation with existing or future surveys in various wavelengths. Such an approach will be extremely useful for studies based on ongoing SZ surveys such as Planck, SPT, and ACT, and upcoming optical surveys such as PanSTARRS ${ }^{2}$ and DES ${ }^{3}$. In addition, as the MCXC provides a self-consistently estimated measure of the total mass $M_{500}$, it can be used to predict various global quantities of the clusters contained within. For example, combination with the appropriate scaling laws allows prediction of the integrated SZ flux $Y_{\mathrm{SZ}, 500}$, or total optical light. In this context, the meta-catalogue will be useful for the defintion of subsamples for deeper analysis, including archival investigations and follow-up observations with SZ and optical/IR instruments, and for a first characterisation of known objects detected in new surveys. However, while the MCXC comprises virtually all known X-ray clusters, its selection function is impossible to reconstruct as the individual selection functions of the subsamples used in its construction are extremely complex and, in most cases, are not known or not available. Use of the MCXC for e.g., $\mathrm{X}$-ray scaling relation studies is thus possible only via the definition of subsamples for which the selection function is known or can be characterised.

The paper is organised as follows. In Sect. 2 we describe the basic properties of the catalogues used to construct the MCXC. In Sect. 3 we explain how the information is homogenised and detail the quantities provided by the MCXC. The handling of duplicate entries is presented in Sect. 4 and in Sect. 5 we discuss various aspects of the final catalogue. In Sect. 6 we summarise our results and present our conclusions.

As a cosmological model we adopt a $\Lambda$ CDM cosmology with $H_{0}=70 \mathrm{~km} \mathrm{~s}^{-1} \mathrm{Mpc}^{-1}, \Omega_{\mathrm{M}}=0.3$ and $\Omega_{\Lambda}=0.7$ throughout the paper. The quantity $h(z)$ is the ratio of the Hubble constant at redshift $z$ to its present value, $H_{0}$, i.e., $h(z)^{2}=\Omega_{\mathrm{m}}(1+$ $z)^{3}+\Omega_{\Lambda}$. All quantities related to the MCXC electronic Table are given in typewriter typeface.

\section{Input X-ray catalogues}

In the following we describe the input catalogues used to construct the MCXC. We recall the basic characteristics of the X-ray surveys used to construct each catalogue and how the X-ray quantities adopted in our work are measured. We discuss only $\mathrm{X}$-ray information essential to the MCXC and focus on the quantities that allow us to compute the luminosities, $L_{500}$. For more details on the individual surveys, and in particular the associated optical observations/follow-up, we refer the reader to the cited papers and references therein.

Generally speaking, two types of X-ray survey can be distinguished: contiguous area surveys, which use data from the ROSAT All-Sky Survey (RASS, Voges et al. 1999), and serendipitous cluster surveys, which are based on data from deeper pointed X-ray observations. In the following, we therefore distinguish between RASS-based and serendipitous catalogues. In addition to handling duplicate entries and removing particular clusters as discussed below, we exclude clusters with

\footnotetext{
2 http://pan-starrs.ifa.hawaii.edu

3 http://www. darkenergysurvey.org
} 
non measured redshifts or luminosities. Table 1 summarises the contributions of the various input catalogues to the MCXC.

The bulk of the X-ray data used to construct the MCXC are derived from ROSAT observations. Exceptions are EMSS and some physical quantities for MACS, as described in more detail below. Future work will include incorporation of as-yet unpublished catalogues such as RDCS (the ROSAT Deep Cluster Survey, Rosati et al. 1998), XCS (the XMM Cluster Survey, Romer et al. 2001), XDCP (the XMM-Newton Distant Cluster Project, Fassbender 2007), and the complete MACS catalogue (Ebeling et al. 2001).

\subsection{RASS-based catalogues}

We compiled data from nine RASS-based contiguous area surveys, as described below.

\subsubsection{REFLEX and NORAS}

REFLEX (ROSAT-ESO Flux Limited X-ray Galaxy Cluster Survey, Böhringer et al. 2004a) is based on RASS data for a survey area covering the southern sky up to a declination $\delta=2.5 \mathrm{deg}$ with the galactic plane $(|b| \leq 20 \mathrm{deg})$ and the regions of the Magellanic clouds excluded. The total survey area is $13924 \mathrm{deg}^{2}$ and the survey is flux-limited (0.1-2.4 keV band flux $\geq 3 \times 10^{-12} \mathrm{erg} \mathrm{s}^{-1} \mathrm{~cm}^{-2}$ ).

NORAS (Northern ROSAT All-Sky galaxy cluster survey Böhringer et al. 2000a) is also based on RASS data excluding the same region around the galactic plane, but covers the northern sky. This survey catalogue is not flux-limited and selection is based on minimum count rate $(0.06 \mathrm{cts} / \mathrm{s}$ in the $0.1-2.4 \mathrm{keV}$ band) and a source extent likelihood.

The data analysis and catalogue production for both NORAS and REFLEX are performed by essentially the same authors and, although REFLEX has been more extensively studied and characterised than NORAS, the information provided is extremely similar. A growth curve analysis is adopted to determine source fluxes (the typical flux measurement accuracy is $10-20$ per cent) and luminosities. The REFLEX catalogue provides aperture luminosities $L_{\mathrm{ap}}$ as well as total luminosities. The latter are computed by estimating the missing flux outside the detection aperture by assuming a $\beta$-model with $\beta=2 / 3$, a core radius $r_{\mathrm{c}}$ which scales with mass, and a cluster extent of $12 \times r_{\mathrm{c}}$. For the NORAS clusters a similar procedure is performed, but the resulting total luminosities are not reported. Therefore the NORAS catalogue provides only aperture luminosities.

For both catalogues data (Böhringer et al. 2000b, 2004b) are retrieved from VizieR ${ }^{4}$. Because of the homogeneity of these two catalogues we merge them into a single NORAS/REFLEX catalogue. The names NORAS and REFLEX are kept as subcatalogue labels. Because of the overlap of the NORAS and REFLEX survey areas, there are ten duplicate entries. For these ten clusters the information provided by NORAS and REFLEX is almost identical and we exclude, for each of the duplicates, the cluster with the larger flux uncertainty.

Since the number of clusters in the combined NORAS/REFLEX catalogue is large (889 objects, see Table 1) and because the information provided by the authors is homogeneous and detailed, it is the cornerstone of the MCXC.

\footnotetext{
${ }^{4}$ http://VizieR.u-strasbg.fr/viz-bin/VizieR
}

\subsubsection{ROSAT BCS and eBCS}

The ROSAT BCS (The ROSAT Brightest Cluster Sample, Ebeling et al. 1998) comprises the brighter sources of the NORAS survey. We use data for the 90 per cent complete BCS, a flux-limited sample $(0.1-2.4 \mathrm{keV}$ band flux $\geq 4.4 \times$ $10^{-12} \mathrm{erg} \mathrm{s}^{-1} \mathrm{~cm}^{-2}$ ) of $z \leq 0.3$ clusters. The $e B C S$ (The extended ROSAT Brightest Cluster Sample, Ebeling et al. 2000a) is the low flux extension of the BCS $(0.1-2.4 \mathrm{keV}$ band flux $\geq 2.8 \times 10^{-12} \mathrm{erg} \mathrm{s}^{-1} \mathrm{~cm}^{-2}$ ).

The type of information provided is the same for both samples. In both cases detection and cluster emission characterisation are based upon the Voronoi tesselation and percolation (VTP) algorithm. The emission outside the detection region is computed by correcting the detected count rate. For clusters this is undertaken by assuming a $\beta$-model profile with $\beta=2 / 3$ and a core radius estimated from the source profile, taking into account the telescope PSF. The resulting total luminosities, the corrected and uncorrected count rates, and the VTP aperture radius are provided. This implies that the luminosity within the VTP aperture radius can be computed for all the clusters in the sample.

Data (Ebeling et al. 2000b,c) are retrieved from VizieR and merged into a single BCS catalogue where the names BCS and eBCS are kept as sub-catalogue labels (see Table 1). There is only one cluster, A1758a, that is listed in both BCS and eBCS. The two luminosities are almost identical and we remove it from the BCS sub-catalogue. In addition, for the Virgo cluster we adopt the luminosity estimate of Böhringer et al. (1994).

\subsubsection{SGP}

The SGP (A Catalog of Clusters of Galaxies in a Region of 1 Steradian around the South Galactic Pole, Cruddace et al. 2002a) covers a region of 1.013 sr centred on the south Galactic pole and is based on the same X-ray source detection and characterisation procedures as REFLEX. The lowest detected flux is $1.5 \times 10^{-12} \mathrm{erg} \mathrm{s}^{-1} \mathrm{~cm}^{-2}$ in the $0.1-2.4 \mathrm{keV}$ band, and a complete subsample can be obtained by imposing a flux limit of $3 \times 10^{-12} \mathrm{erg} \mathrm{s}^{-1} \mathrm{~cm}^{-2}$.

Luminosities are computed within a cutoff radius provided by the growth curve analysis. Since the cutoff radius is not given in the catalogue, we treat the quoted luminosity as the total luminosity.

Data for the entire non-flux-limited, SGP sample (Cruddace et al. 2002b, 2003) were retrieved from VizieR.

\subsubsection{NEP}

The NEP (The ROSAT North Ecliptic Pole survey, Henry et al. 2006) surrounds the north ecliptic pole in a survey area of $80.6 \mathrm{deg}^{2}$, and has the deepest exposure in the northern RASS (exposure times from 2000 to over $40000 \mathrm{~s}$ ). Source detection is based on Voges et al. (1999) and the selection is performed by adopting thresholds for the source extent likelihood and signalto-noise ratio.

The quoted total luminosities are computed from size corrected fluxes. The latter are computed from detected fluxes within apertures of radius $5^{\prime}\left(6.5^{\prime}\right.$ for RXJ1834.1+7057) by assuming a PSF-corrected $\beta$-profile with $\beta=2 / 3$ and a fixed core radius of $180 \mathrm{kpc}$. The profile is integrated up to $R_{200}$, which is estimated from the size-temperature relation of Evrard et al. (1996). Size correction factors are provided so that aperture luminosities can be computed. 
Table 1. Number of clusters in the catalogues used to construct the MCXC before and after handling of multiple entries.

\begin{tabular}{|c|c|c|}
\hline Catalogue & No. of clusters & No. of clusters \\
\hline Sub-catalogues & Input & MCXC \\
\hline NORAS/REFLEX & 889 & 878 \\
\hline NORAS & 445 & 437 \\
\hline REFLEX & 444 & 441 \\
\hline $400 S D$ & 266 & 257 \\
\hline 400SD_SER & 242 & 237 \\
\hline 400SD_NONSER & 24 & 20 \\
\hline $160 S D$ & 199 & 90 \\
\hline$B C S$ & 312 & 80 \\
\hline BCS & 205 & 47 \\
\hline eBCS & 107 & 33 \\
\hline$S G P$ & 157 & 55 \\
\hline SHARC & 69 & 29 \\
\hline SHARC_BRIGHT & 37 & 14 \\
\hline SHARC_SOUTH & 32 & 15 \\
\hline WARPS & 159 & 78 \\
\hline WARPS & 34 & 11 \\
\hline WARPSII & 125 & 67 \\
\hline$N E P$ & 63 & 48 \\
\hline$M A C S$ & 51 & 39 \\
\hline MACS_MJFV & 23 & 18 \\
\hline MACS_BRIGHT & 22 & 15 \\
\hline MACS_DIST & 6 & 6 \\
\hline$C I Z A$ & 130 & 128 \\
\hline CIZAI & 73 & 72 \\
\hline CIZAII & 57 & 56 \\
\hline EMSS & 102 & 61 \\
\hline EMSS_1994 & 81 & 47 \\
\hline EMSS_2004 & 21 & 14 \\
\hline TOTAL & 2397 & 1743 \\
\hline
\end{tabular}

Data for the whole flux-limited sample (0.5-2 keV band flux $\geq 2$. $\times 10^{-14} \mathrm{erg} \mathrm{s}^{-1} \mathrm{~cm}^{-2}$, Henry et al. 2006) were retrieved from VizieR, and only sources identified as clusters were selected (see Table 1).

\subsubsection{MACS}

The MACS (Massive Cluster Survey, Ebeling et al. 2001) is based on the ROSAT Bright Source Catalogue with the aim of increasing the number of known very luminous, $z \geq 0.3$ clusters. A MACS catalogue has not yet been published in its entirety and we therefore collected data from different publications as detailed below. We would like to point out that the data reported in these publications are based on Chandra follow-up observations and that these publications yield all publicly-available MACS clusters with coordinates, redshifts, and luminosities (i.e., the minimal set of quantities required for the MCXC).

Properties of a complete subsample of $z>0.5$ MACS clusters (the MACS_DIST sub-catalogue, twelve objects) are listed in Ebeling et al. (2007). A further complete subsample of bright objects in the $0.3<z<0.5$ redshift range (the MACS_BRIGHT sub-catalogue, 34 clusters) are given in Ebeling et al. (2010).
All MACS_BRIGHT clusters are analysed in more detail in Mantz et al. (2010b), where quantities such as $L_{500}, M_{500}$, etc., are provided. This information is also merged into the MACS_BRIGHT sub-catalogue.

Further MACS clusters are analysed in Maughan et al. (2008, the MACS_MJFV sub-catalogue, 23 objects), who provide very complete information on the physical properties of these objects. Of the MACS_MJFV sample there are six clusters in common with the MACS_DIST sub-catalogue and twelve clusters in common with the MACS_BRIGHT sub-catalogue. We construct a unique MACS catalogue by merging the three sub-catalogues and keeping only measurements given by Maughan et al. (2008) for the eighteen duplicate clusters (see Table 1). Apart from the six MACS_DIST luminosity measurements in Ebeling et al. (2007), the luminosities $L_{500}$ are directly available for all MACS clusters.

\subsection{6. $\mathrm{CIZA}$}

The CIZA (Clusters in the Zone of Avoidance, Ebeling et al. 2002; and Kocevski et al. 2007, respectively CIZAI and CIZAII) catalogues are based on the ROSAT Bright Source Catalogue and focus on the region around the galactic plane ( $|b| \leq 20 \mathrm{deg})$. Candidate selection is based on limits on the detected fluxes and spectral hardness ratios. CIZAI comprises the X-ray brightest objects (flux $\geq 5$. $\times 10^{-12} \mathrm{erg} \mathrm{s}^{-1} \mathrm{~cm}^{-2}$ ), while CIZAII is its lowflux extension (flux $\geq 3 . \times 10^{-12} \mathrm{erg} \mathrm{s}^{-1} \mathrm{~cm}^{-2}$ ).

Quoted luminosities are computed from raw RASS data using very large apertures and can be therefore safely interpreted as total luminosities.

The type of data available for the two catalogues is identical, and after retrieving data (Ebeling et al. 2002; Kocevski et al. 2007) from VizieR, we merged them into a single CIZA catalogue where the names CIZAI and CIZAII define the subcatalogues (see Table 1).

\subsection{Serendipitous catalogues}

We compiled data from a further seven serendipitous surveys as described below.

\subsection{1. $160 \mathrm{SD}$}

The 160SD (The 160 Square Degree ROSAT Survey, Mullis et al. 2003) is based on the serendipitous detection of extended X-ray emission in 647 archival ROSAT PSPC observations. With the galactic plane $(|b| \leq 30 \mathrm{deg})$ and the regions of the Magellanic clouds excluded, the resulting sky coverage at high fluxes is $160 \mathrm{deg}^{2}$.

A wavelet algorithm is used to detect galaxy clusters and the quoted total luminosities are computed from the detected fluxes by assuming a $\beta$-profile with $\beta=2 / 3$ and a fitted core radius.

We retrieved the full dataset (Mullis et al. 2003) from VizieR and selected only sources identified as galaxy clusters.

\subsection{2. $400 \mathrm{SD}$}

The 400SD (The 400 Square Degree ROSAT PSPC Galaxy Cluster Survey, Burenin et al. 2007) extends the 160SD methodology to additional PSPC observations by adopting less restrictive selection criteria (e.g., galactic latitude and absorption, exposure times). A total of 1610 fields, corresponding to a total survey area of $397 \mathrm{deg}^{2}$, are analysed to yield a large 
flux-limited $\left(0.5-2 \mathrm{keV}\right.$ band flux $\left.\geq 1.4 \times 10^{-13} \mathrm{erg} \mathrm{s}^{-1} \mathrm{~cm}^{-2}\right)$ cluster catalogue. 400SD data is available for serendipitously and not entirely serendipitously detected clusters (clusters at redshift very close to the target redshift).

We retrieved data (Burenin et al. 2009) from VizieR, and merged the information into a unique 400SD catalogue, introducing the sub-catalogue labels 400SD_SER and 400SD_NONSER to distinguish between the two classes of objects (see Table 1).

\subsubsection{SHARC Bright and SHARC Southern}

The SHARC survey is based on archival ROSAT PSPC observations. The SHARC Bright (Bright Serendipitous High-Redshift Archival ROSAT Cluster survey, Romer et al. 2000a) is a wide area shallow survey covering a total area of $178.6 \mathrm{deg}^{2}$ with a flux limit of $1.63 \times 10^{-13} \mathrm{erg} \mathrm{s}^{-1} \mathrm{~cm}^{-2}$. The SHARC Southern (The Southern Serendipitous High-Redshift Archival ROSAT Cluster survey, Burke et al. 2003a) is a narrow area deep survey covering $17.7 \mathrm{deg}^{2}$ with a flux limit of $4.66 \times 10^{-14} \mathrm{erg} \mathrm{s}^{-1} \mathrm{~cm}^{-2}$. Cluster detection is based on a wavelet and sliding-box techniques, respectively.

For both catalogues a $\beta$-profile with fixed $\beta=2 / 3$ and $r_{\mathrm{c}}$ is used to determine the total luminosity and a circular aperture of radius $r_{80}$, which contains 80 per cent of the total flux. This implies that in addition to the extrapolated total luminosities, the aperture luminosities $L_{\text {ap }} \equiv L\left(<r_{80}\right)$ are available.

Data (Burke et al. 2003b; Romer et al. 2000b) are retrieved from VizieR and merged into a single SHARC catalogue (only sources identified as clusters are selected from Romer et al. 2000b) with sub-catalogues labelled SHARC_SOUTH and SHARC_BRIGHT (see Table 1).

\subsubsection{WARPS and WARPSII}

The WARPS survey is also based on ROSAT PSPC observations. WARPS (Wide Angle ROSAT Pointed Survey, Perlman et al. 2002a), covers $16.2 \mathrm{deg}^{2}$ in 86 PSPC fields, while its extension WARPSII (Wide Angle ROSAT Pointed Survey II, Horner et al. 2008) covers $56.7 \mathrm{deg}^{2}$ in 301 PSPC fields. The WARPS survey uses the VTP algorithm for cluster detection and characterization.

The quoted total luminosities are computed as in Ebeling et al. (1998), but no information which allows the computation of aperture luminosities is reported.

Data (Perlman et al. 2002b; Horner et al. 2009) are retrieved from VizieR. For both catalogues we include clusters below the nominal flux limit that defines the statistically complete sample. The two catalogues are merged into a single WARPS catalogue and WARPSI and WARPSII are adopted as sub-catalogue labels (see Table 1).

\subsubsection{EMSS}

The EMSS (Einstein Observatory Extended Medium Sensitivity Survey, Gioia et al. 1990) cluster catalogue is constructed from a flux-limited sample of sources serendipitously detected in Einstein IPC (Imaging Proportional Counter) fields at high galactic latitudes.

Data are compiled from the Tables published in Gioia \& Luppino (1994) and Henry (2004). While the sample presented in Gioia \& Luppino (1994) is the most complete and up-to-date work on the entire EMSS cluster catalogue, Henry (2004) provides more reliable ASCA measurements for the $z \geq 0.3$ EMSS clusters. The Einstein luminosities reported in Gioia \& Luppino (1994) are computed from the flux measured in a $2.4 \times 2 ! 4$ detection cell by adopting a $\beta$-model with fixed $\beta=2 / 3$. The information provided is not sufficient to compute aperture luminosities from the quoted total luminosities. The ASCA luminosities in Henry (2004) are total luminosities. Since distant clusters are not resolved by ASCA, these luminosities were derived by assuming that the clusters are point sources. Hence in this case only total luminosities are available.

Clusters in the Henry (2004) sample are removed from Gioia \& Luppino (1994) $)^{5}$. We remove MS1209.0+3917, MS1333.3+1725, and MS1610.4+6616 for the reasons mentioned in Henry (2004). The data are then merged into a single EMSS catalogue where the names EMSS_1994 and EMSS_2004 denote the sub-catalogue labels (see Table 1).

\section{Data extraction and homogenisation}

The data provided by the different input catalogues (positions, redshifts, names, luminosities, etc.) are rather similar. However some data homogenisation is needed, in particular for quantities such as luminosity and mass.

As detailed above, in many cases the luminosity is measured within some small aperture $R_{\text {ap }}$, where $L_{\text {ap }} \equiv L\left(<R_{\text {ap }}\right)$ is the corresponding aperture luminosity, and then extrapolated to some larger radius using a reasonable model of the surface brightness profile. Extrapolation might be performed at a very large radius, implying that the derived luminosity is basically equal to the total luminosity $L_{\mathrm{tot}}=L(<\infty)$. With the present generation of X-ray observations, the standard choice is $R_{500}$ $\left(L_{500} \equiv L\left(<R_{500}\right)\right)$, and we have chosen this radius for the MCXC data homogenisation procedure.

The assumed cosmological model is of course at the basis of our homogenisation procedure. In the following all luminosities and other cluster parameters which depend on the distance scale are converted to our reference cosmology (i.e. $\Omega_{\mathrm{M}}=0.3, \Omega_{\Lambda}=$ 0.7 , and $H_{0}=70 \mathrm{~km} \mathrm{~s}^{-1} \mathrm{Mpc}^{-1}$ ).

Below we list all the quantities that are provided by the MCXC and explain in detail how they are derived from the original information in the input catalogues. The names of the quantities as given in the associated electronic Table are given in typewriter typeface. MCXC clusters are ordered by right ascension. As an example we list the first 40 entries in Table 2.

\subsection{Coordinates and redshifts}

The cluster coordinates given in the input catalogues are those of the cluster centroid determined from X-ray data (apart from those in the sub-catalogue EMSS_1994 which are the coordinates of the cluster optical position). For the MCXC, all coordinates are converted to right ascension and declination for the epoch J2000 in hours (degrees), minutes, and seconds (RAJ2000 and DEJ2000) and in units of decimal degrees (_RAJ2000 and _DEJ2000). We also provide the cluster positions in galactic coordinates - GLON and GLAT are galactic longitude and latitude, respectively, in degrees (see Table 2).

No manipulation is needed for the cluster redshifts $z$. As stated above, only clusters with measured redshift are retained in the MCXC (see Table 2). In Fig. 1, we show the redshift histograms of the individual input catalogues used to construct the MCXC, and of the MCXC after handling of multiple entries (see

5 For a comparison between EMSS and ASCA flux measurements see Henry (2004). 

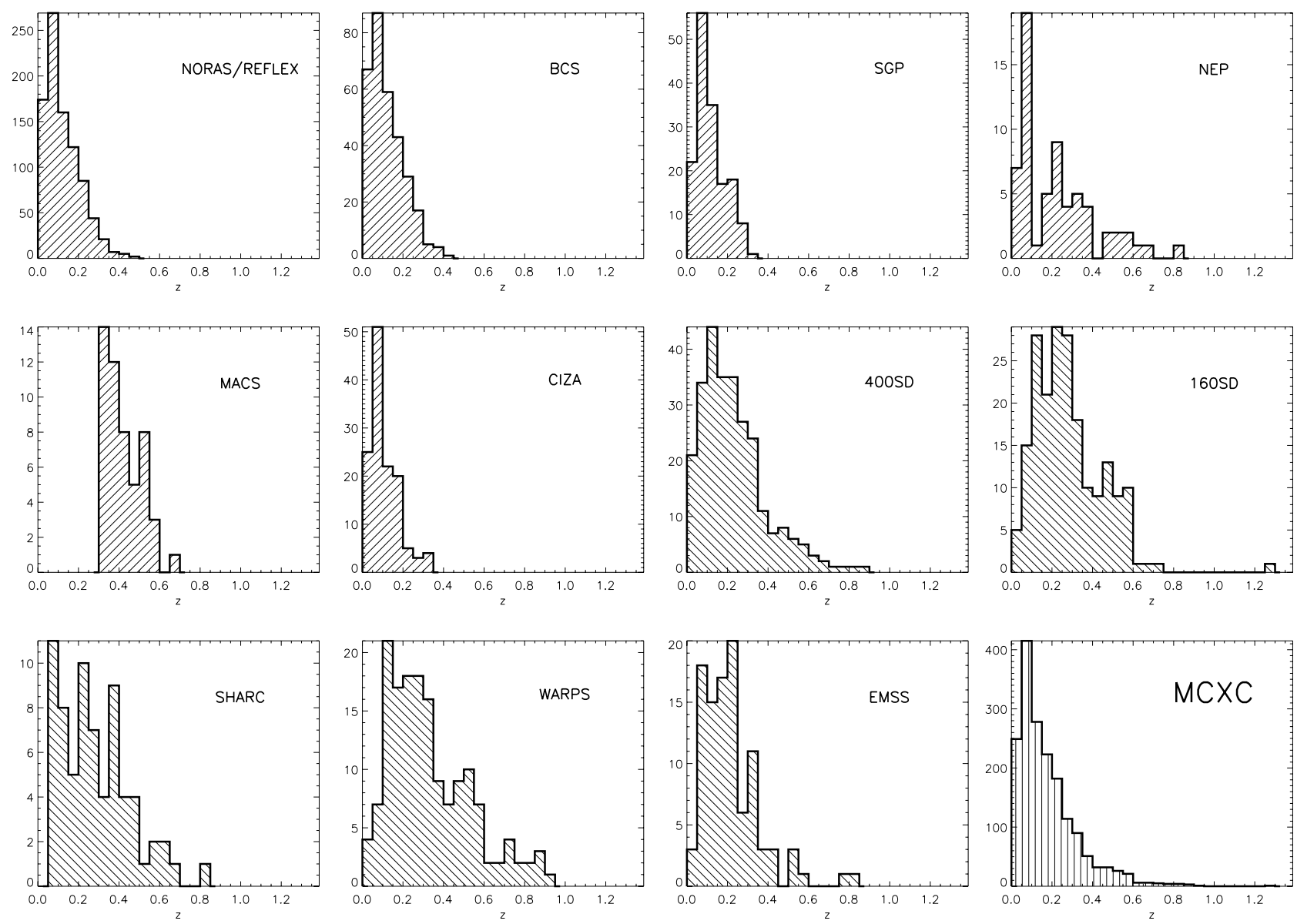

Fig. 1. Redshift histograms of the input catalogues used to construct the MCXC and of the MCXC after handling of multiple entries. Different area shadings are used for RASS-based, serendipitous, and MCXC catalogues.

Sect. 4). The histograms highlight the different redshift ranges typically probed by serendipitous and RASS-based surveys, with the latter generally being confined to local and medium redshift clusters.

\subsection{Names}

Two types of cluster name are usually listed in the input catalogues: the name assigned by the authors NAME, and the alternative name NAME_ALT (see Table 2). NAME is usually constructed from the cluster coordinates (e.g., RXJ0041.1-2339 in 160SD, MS0007.2-3532 in EMSS, MACSJ0011.7-1523 in MACS, RXC J0000.1+0816 in NORAS/REFLEX, CIZA, and SGP, RX J1716.6+6410 in NEP and SHARC, J0022.0+0422 in WARPS). Exceptions to this format are BCS and 400SD. BCS names are listed as they appear in optical catalogues (e.g. ZwC11432, A602), while in the 400SD, names are not assigned. We therefore assigned a NAME to 400 SD_SER clusters according to the standard SIMBAD 6 format acronym "BVH2007 NNN" (Burenin+Vikhlinin+Hornstrup+, 2007, e.g., BVH2007 193), and for the 400SD_NONSER we created a new acronym "BVH2007 NS NNN" (e.g., BVH2007 NS 12). For all but the 400SD clusters we retain the original names as listed in the input catalogues.

Alternative names in the input catalogues are mostly based on catalogued optical counterparts to the X-ray sources: Abell

\footnotetext{
${ }^{6}$ http://simbad.u-strasbg.fr/simbad/
}

and Zwicky Cluster names, names of NGC and UGC central dominant galaxies, etc. (e.g., A2894, ZwCl 0104.9+5350, UGC 12890). Thus in some cases alternative names refer to objects that are not clusters of galaxies. In other cases alternative names are given as notes or comments, and we also use this information to construct NAME_ALT in the MCXC by extracting the suitable piece of information. Alternative names are homogenised so as to match both SIMBAD and NED ${ }^{7}$ standards. When this is not possible we choose the SIMBAD acronym conventions. Moreover, when multiple alternative names are available, they are listed separated with a comma. For BCS clusters we set NAME_ALT equal to NAME. For 160SD and 400SD clusters alternative names are extracted from the notes. For 160SD clusters the identifier "VMF98 NNN" is also used. In most of the input catalogues alternative names end with letters for double systems (e.g., A2384 (A), A3574E, etc.). Such information is important because it indicates whether the measured luminosity refers to the whole system to only a part of it.

Our choice of formats for NAME and NAME_ALT in the MCXC is made in order to facilitate queries in the SIMBAD and NED databases. Both NAME and NAME_ALT also facilitate the handling of duplicate entries as discussed extensively below in Sect. 4.

As NAME and NAME_ALT are heterogeneous and do not always exist in the original catalogues, a homoegenous designation of the clusters, which follows the IAU designation rules, is added in the first column (NAME_MCXC in Table 2). This internal

7 http://nedwww.ipac.caltech.edu/ 


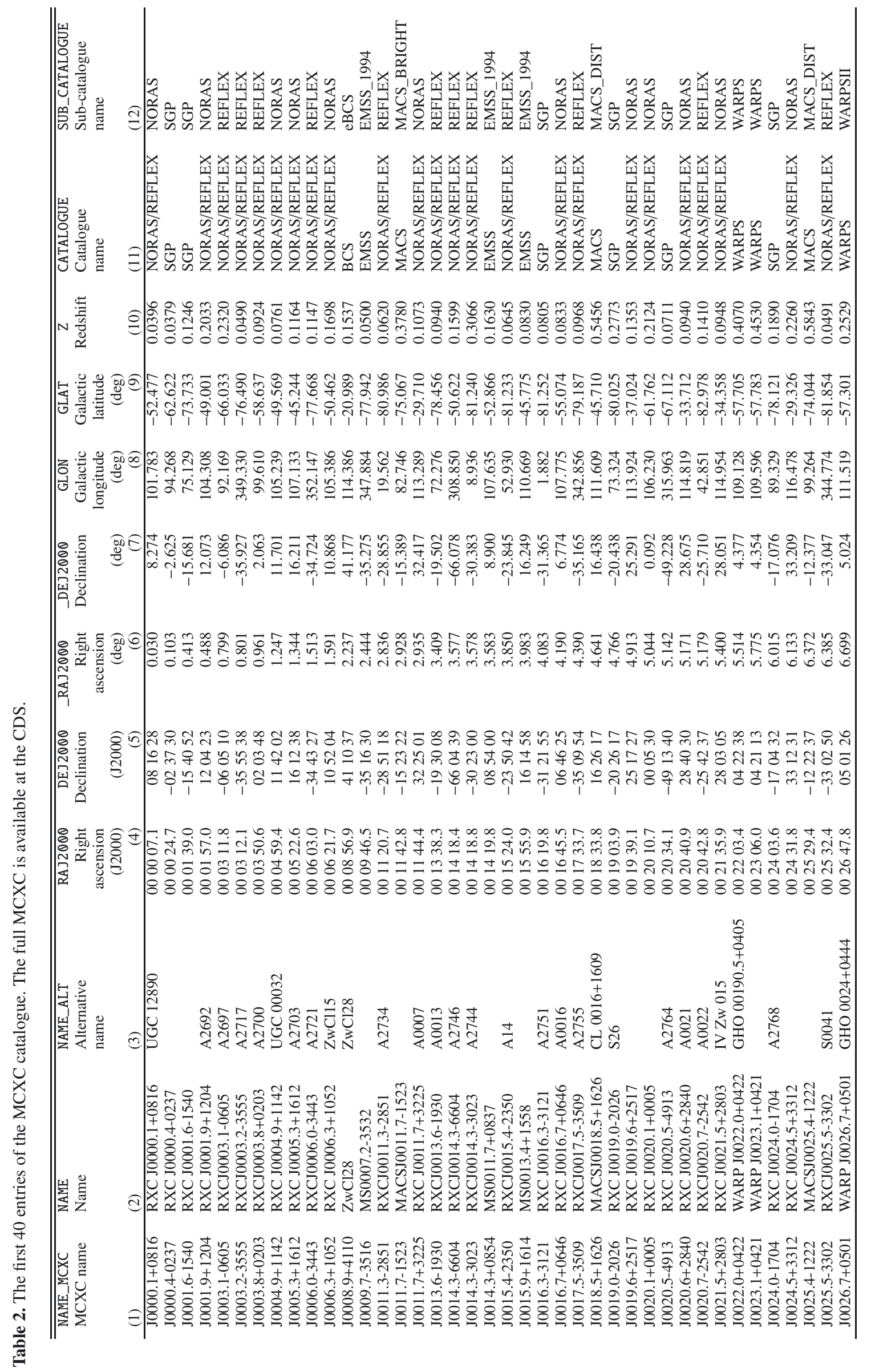


Table 2. continued.

\begin{tabular}{|c|c|c|c|c|c|c|c|}
\hline $\begin{array}{l}\text { NAME_MCXC } \\
\text { MCXC name }\end{array}$ & $\begin{array}{l}\text { SCALE } \\
\text { Scale } \\
\text { (kpc/") } \\
(13) \\
\end{array}$ & $\begin{array}{l}L_{-} 500 \\
L_{500} \\
\left(10^{44} \mathrm{erg} \mathrm{s}^{-1}\right) \\
(14)\end{array}$ & $\begin{array}{l}M_{1500} \\
M_{500} \\
\left(10^{14} M_{\odot}\right) \\
(15)\end{array}$ & $\begin{array}{l}\text { R_500 } \\
R_{500} \\
(\mathrm{Mpc}) \\
(16) \\
\end{array}$ & $\begin{array}{l}\text { NOTES } \\
\text { Notes } \\
(17) \\
\end{array}$ & $\begin{array}{l}\text { CAT_OV } \\
\text { Catalogues } \\
\text { overlap } \\
\text { (18) } \\
\end{array}$ & $\begin{array}{l}\text { L_500_RAT } \\
L_{500} / L_{500, M C X C} \\
(19) \\
\end{array}$ \\
\hline $\mathrm{J} 0000.1+0816$ & 0.784 & 0.196 & 0.737 & 0.630 & & $\mathrm{BCS}$ & 1.084 \\
\hline J0000.4-0237 & 0.752 & 0.052 & 0.330 & 0.482 & & & \\
\hline J0001.6-1540 & 2.234 & 0.815 & 1.656 & 0.802 & & & \\
\hline J0001.9+1204 & 3.342 & 1.990 & 2.693 & 0.918 & & & \\
\hline J0003.1-0605 & 3.698 & 6.107 & 5.219 & 1.133 & & SGP & 0.952 \\
\hline J0003.2-3555 & 0.959 & 0.442 & 1.202 & 0.739 & losStr & SGP & 0.886 \\
\hline $\mathrm{J} 0003.8+0203$ & 1.719 & 0.847 & 1.734 & 0.823 & & eBCS $\mid$ SGP & $0.920 \mid 0.922$ \\
\hline J0004.9+1142 & 1.443 & 0.519 & 1.301 & 0.752 & & eBCS & 0.967 \\
\hline $\mathrm{J} 0005.3+1612$ & 2.107 & 1.579 & 2.493 & 0.922 & B & EMSS_1994 & 0.533 \\
\hline J0006.0-3443 & 2.080 & 1.809 & 2.712 & 0.949 & & SGP & 0.949 \\
\hline $\mathrm{J} 0006.3+1052$ & 2.895 & 2.273 & 2.994 & 0.962 & & eBCS & 0.933 \\
\hline $\mathrm{J} 0008.9+4110$ & 2.668 & 2.111 & 2.896 & 0.957 & & & \\
\hline J0009.7-3516 & 0.977 & 0.262 & 0.873 & 0.664 & & & \\
\hline J0011.3-2851 & 1.195 & 1.086 & 2.061 & 0.881 & losStr & SGP & 0.914 \\
\hline J0011.7-1523 & 5.188 & 8.900 & 7.200 & 1.190 & & & \\
\hline $\mathrm{J} 0011.7+3225$ & 1.962 & 2.572 & 3.378 & 1.023 & & BCS & 1.042 \\
\hline J0013.6-1930 & 1.746 & 1.236 & 2.182 & 0.888 & losStr & SGP & 0.955 \\
\hline J0014.3-6604 & 2.756 & 2.827 & 3.446 & 1.012 & $\mathrm{X}$ & & \\
\hline J0014.3-3023 & 4.522 & 11.818 & 7.361 & 1.236 & & SGP|MACS_BRIGHT & $0.985 \mid 1.139$ \\
\hline J0014.3+0854 & 2.800 & 1.928 & 2.722 & 0.934 & & & \\
\hline J0015.4-2350 & 1.240 & 0.326 & 0.988 & 0.689 & $\mathrm{X}$ & SGP & 0.956 \\
\hline J0015.9+1614 & 1.561 & 0.320 & 0.964 & 0.679 & & & \\
\hline J0016.3-3121 & 1.518 & 0.495 & 1.261 & 0.743 & & & \\
\hline J0016.7+0646 & 1.566 & 0.319 & 0.963 & 0.679 & & eBCS & 1.385 \\
\hline J0017.5-3509 & 1.792 & 0.692 & 1.529 & 0.788 & losStr & SGP & 0.964 \\
\hline $\mathrm{J} 0018.5+1626$ & 6.386 & 17.911 & 7.785 & 1.148 & & EMSS_2004 & 0.593 \\
\hline J0019.0-2026 & 4.215 & 5.571 & 4.763 & 1.081 & & & \\
\hline J0019.6+2517 & 2.397 & 1.442 & 2.327 & 0.895 & & & \\
\hline $\mathrm{J} 0020.1+0005$ & 3.458 & 0.687 & 1.398 & 0.735 & & & \\
\hline J0020.5-4913 & 1.356 & 0.268 & 0.873 & 0.659 & & & \\
\hline J0020.6+2840 & 1.746 & 1.435 & 2.389 & 0.916 & & BCS & 1.201 \\
\hline J0020.7-2542 & 2.482 & 2.872 & 3.527 & 1.026 & & SGP & 0.912 \\
\hline $\mathrm{J} 0021.5+2803$ & 1.759 & 0.968 & 1.878 & 0.845 & & BCS & 0.956 \\
\hline $\mathrm{J} 0022.0+0422$ & 5.430 & 0.582 & 1.082 & 0.628 & & & \\
\hline $\mathrm{J} 0023.1+0421$ & 5.781 & 0.785 & 1.250 & 0.647 & & & \\
\hline J0024.0-1704 & 3.156 & 1.484 & 2.276 & 0.872 & & & \\
\hline $\mathrm{J} 0024.5+3312$ & 3.626 & 2.993 & 3.394 & 0.983 & & & \\
\hline J0025.4-1222 & 6.603 & 8.042 & 4.623 & 0.950 & & & \\
\hline J0025.5-3302 & 0.961 & 0.495 & 1.287 & 0.756 & & SGP & 0.929 \\
\hline $\mathrm{J} 0026.7+0501$ & 3.943 & 0.326 & 0.860 & 0.616 & & & \\
\hline
\end{tabular}

designation allows a fully unambiguous cluster identification within the MCXC catalogue. We would like to point out that NAME and NAME_ALT often contain information on how the cluster was discovered (Abell, Zwicky, EMSS, MACS, etc. clusters). This kind of information is not contained in NAME_MCXC.

\subsection{Catalogue and sub-catalogue}

As explained above in Sect. 2 and listed in Table 1, for each cluster the input catalogue and sub-catalogue names are given in CATALOGUE and SUB_CATALOGUE (see Table 2). If no subcatalogue exists the sub-catalogue name is equal to the catalogue name.

\subsection{Luminosities}

\subsubsection{Luminosity calculation}

The luminosities are homogenised according to the following procedure:

1. When necessary, we first convert the input luminosity (e.g., 0.5-2 keV band in NEP, bolometric in MACS_MJFV, $0.3-3.5 \mathrm{keV}$ band in EMSS_1994) to the $0.1-2.4 \mathrm{keV}$ energy band using the MEKAL plasma code (Mewe et al. 1985; Liedahl et al. 1995). The temperature dependence of this conversion is taken into account either by using measured temperatures when available in the input catalogue, or by iteration about the non-core-excised luminosity-temperature relation of Pratt et al. (2009), assuming an abundance of 0.3. In the following all the quoted luminosities are therefore as measured in the $0.1-2.4 \mathrm{keV}$ energy band for our reference cosmology.

2. The resulting luminosities are then converted to $L_{500}$. We adopt two different procedures, depending on the type of luminosity measurement available in the input catalogue.

(a) If only the total luminosity $L_{\text {tot }}$ (i.e., extrapolated up to large distances) is available, we adopt $L_{500}=a \times L_{\text {tot }}$, where $a$ is the ratio $L_{500} / L_{\text {tot }}$ for a luminosity profile model based in the average gas density profile derived from the representative X-ray cluster sample REXCESS (Croston et al. 2008). More precisely, from the individual scaled density profiles (see Arnaud et al. 2010, left panel of Fig. 3), we computed the average profile and fitted it with the AB-model given by Eq. (2) of Pratt \& Arnaud (2002):

$$
\rho_{\text {gas }} \propto\left(\frac{x}{x_{\mathrm{c}}}\right)^{-\alpha} \times\left[1+\left(\frac{x}{x_{\mathrm{c}}}\right)^{2}\right]^{-3 \beta / 2+\alpha / 2}
$$




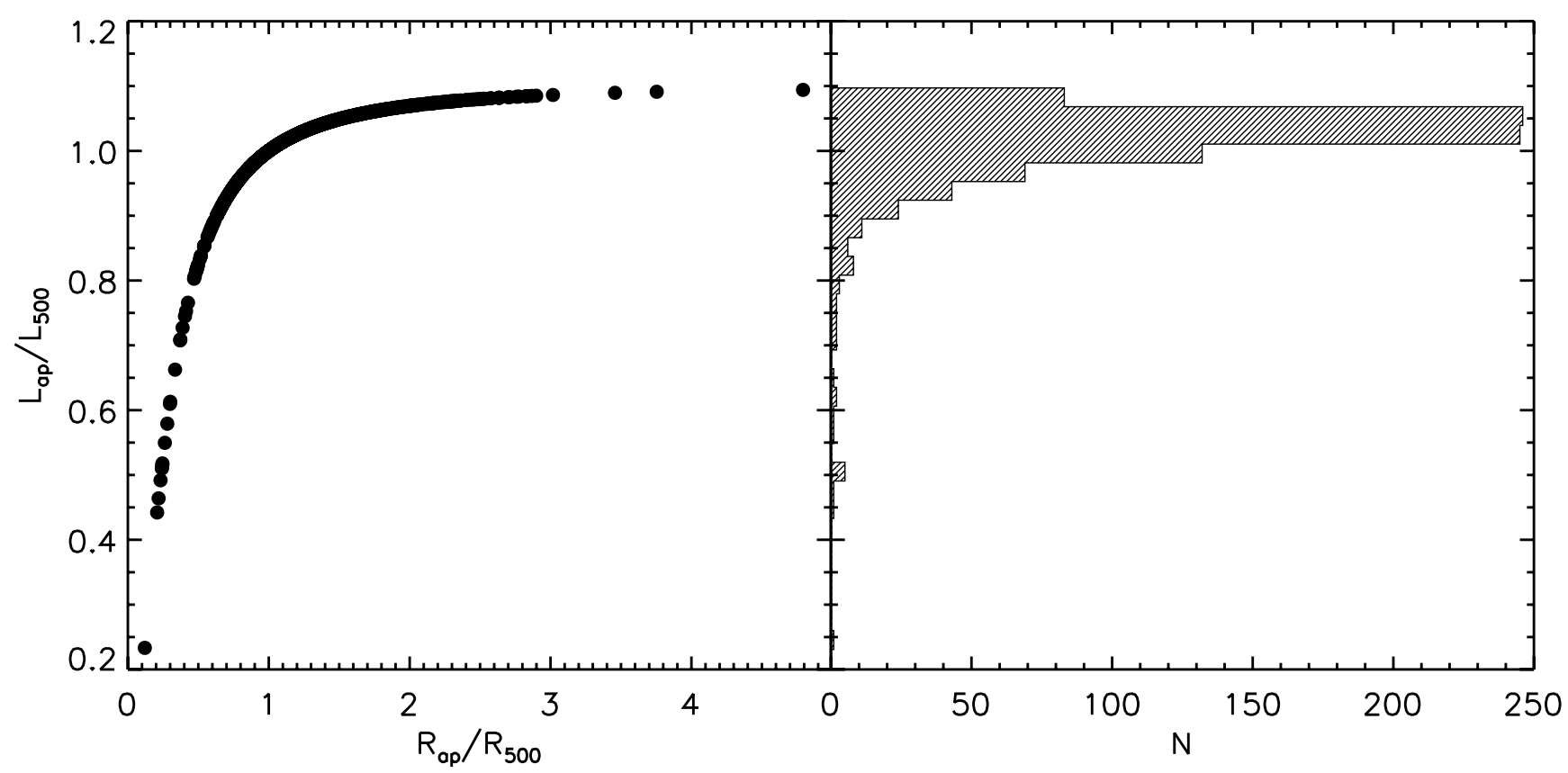

Fig. 2. Relation between input quantities $R_{\text {ap }}$ and $L_{\text {ap }}=L\left(<R_{\text {ap }}\right)$ and the iteratively estimated $R_{500}$ and $L_{500}=L\left(<R_{500}\right)$ for the NORAS/REFLEX clusters. The luminosity ratio as a function of aperture radius ratio is shown in the left panel, while the luminosity ratio histogram is shown in the right panel.

for $x=r / R_{500}$, finding $x_{\mathrm{c}}=0.303, \alpha=0.525$, and $\beta=0.768$. As the $[0.1-2.4] \mathrm{keV}$ band luminosity is only weakly temperature-dependent (e.g., Mewe et al. 1985), and temperature profiles of clusters vary only by a factor of $\sim 2-3$ with radius (e.g., Piffaretti et al. 2005; Pratt et al. 2007), a three-dimensional average luminosity profile is obtained by simply taking the square of the density profile given in Eq. (1).

Since only recently observational progress has shown that the AB-model (Eq. (1)) yields a more accurate description than the traditional $\beta$-model (see Croston et al. 2008 , and references therein), most of the analyses listed in Sect. 2 adopted the latter when extrapolating luminosities to large radii. For the sake of clarity, in Appendix A we illustrate the differences between luminosities computed adopting the AB-model or the $\beta$-model. We notice that the mean REXCESS density profile was defined from a sample with mass $M_{500} \gtrsim 10^{14} M_{\odot}$. The recent analysis of the pressure profiles of galaxy groups with masses as small as $M_{500} \sim 10^{13} M_{\odot}$ by Sun et al. (2011) shows perfect agreement with REXCESS results. Taken together with the good agreement between temperature profiles in group and cluster scale objects (e.g., Rasmussen \& Ponman 2007), and the weak dependence of temperature on radius, this result supports our use of the REXCESS density profile for lower luminosity systems.

As the observed luminosities are derived from integration of surface brightness profiles within circular apertures, the three dimensional average luminosity profile is cylindrically integrated up to aperture radii of 1 and $5 \times R_{500}$ to compute $L_{500}$ and $L_{\mathrm{tot}}$, respectively. The cluster boundary is also assumed to be equal to $5 \times R_{500}$. We find a ratio $a=L_{500} / L_{\text {tot }}=0.91$ and that the exact choice of the aperture enclosing the total luminosity is not relevant. This constant $\sim 10$ per cent correction is therefore applied to all the 160SD, 400SD, SGP, WARPS, CIZA, MACS_DIST, and EMSS clusters for which only total luminosities are available (see Sect. 2). For the MACS_MJFV and MACS_BRIGHT clusters no conversion is needed since the quoted luminosities are $L_{500}$.

(b) For the remaining clusters, i.e. those with available aperture luminosities $L_{\mathrm{ap}}$ (1333 objects in total), we compute $L_{500}$ iteratively as follows.

The basic ingredients of this iterative procedure are the three dimensional luminosity profile model computed from Eq. (1) as detailed above and the luminosity-mass relation ( $L-M$ relation, hereafter):

$h(z)^{-7 / 3}\left(\frac{L_{500}}{10^{44} \mathrm{erg} \mathrm{s}^{-1}}\right)=C\left(\frac{M_{500}}{3 \times 10^{14} M_{\odot}}\right)^{\alpha}$,

with $\log (C)=0.274$ and $\alpha=1.64$ (see Table 1 in Arnaud et al. 2010). These values are slightly different from those given in Pratt et al. (2009) due to Arnaud et al.'s use of an updated $M_{500}-Y_{\mathrm{X}}$ relation. Specifically, we use the relation in Eq. (2) of Arnaud et al. (2010), i.e. we adopt a non-self-similar slope for the $M_{500}-Y_{\mathrm{X}}$ relation. The adopted $C$ and $\alpha$ values are derived from REXCESS luminosity data uncorrected for the Malmquist bias. The effect of these choices is further discussed below.

For a given $M_{500}$ estimated using Eq. (2), the characteristic radius $R_{500}$ entering into Eq. (1) is computed using:

$M_{500}=\frac{4 \pi}{3} R_{500}^{3} 500 \rho_{\mathrm{c}}(z)$,

where the critical density is $\rho_{\mathrm{c}}(z)=3 H(z)^{2} / 8 \pi G$. 
For a given cluster with measured luminosity $L_{500}$, the set of Eqs. (1)-(3) allow us to fully model the observed radial luminosity profile. Cylindrical integration is needed to relate the three dimensional luminosity profile to the observed one. In practice, the equations are used to iteratively compute $L_{500}$ from $R_{\text {ap }}$ and $L_{\text {ap }}=L\left(<R_{\text {ap }}\right)$ (the circular aperture radius and the aperture luminosity, respectively). The latter are either directly available from the input catalogues or can be computed as explained in Sect. 2 for the NORAS/REFLEX, BCS, SHARC, and NEP catalogues. The iteration adopts an arbitrary starting value for the luminosity $L_{500}$ in Eq. (2), which yields an estimated $R_{500}$ through Eq. (3), and thus an observed luminosity profile which is then normalised such that $L_{\mathrm{ap}}=L\left(<R_{\mathrm{ap}}\right)$. The resulting profile is used to compute $L_{500}=L\left(<R_{500}\right)$, which is subsequently adopted as a new value for the luminosity $L_{500}$ in Eq. (2). The iteration converges rapidly and obviously the final value of $L_{500}$ does not depend on the adopted starting luminosity.

In Fig. 2 we illustrate the relation between the input luminosity $L_{\text {ap }}$ and $L_{500}$ for the NORAS/REFLEX clusters. As expected, small/large apertures yield final luminosities $L_{500}$ which are higher/lower than the input aperture luminosities (left panel). While on average the difference between $L_{\mathrm{ap}}$ and $L_{500}$ is $\sim 5$ per cent, it is very relevant for a significant portion of the sample (right panel).

Using the two above-described procedures we can therefore systematically compute $L_{500}$, the $0.1-2.4 \mathrm{keV}$ energy band luminosities within $R_{500}$, for all the clusters (Table 2, L_500).

In the literature the luminosity is sometimes extrapolated within $R_{200}$. We compute the ratio $L_{500} / L_{200}$ in the same way as we compute the ratio $L_{500} / L_{\text {tot }}$ when aperture luminosities are not available (i.e., assuming AB-model Eq. (1) in procedure (a) above), adopting $R_{200} / R_{500}=1.52$. The latter value is computed assuming an NFW profile with concentration $c_{200}=$ 4.61, the mean concentration measured for a morphologically relaxed cluster sample by Pointecouteau et al. (2005). We find $L_{500} / L_{200}=0.96$. A fair estimate of the luminosity $L_{200}=$ $L\left(<R_{200}\right)$ can thus be obtained from the listed $L_{500}$ by assuming this average luminosity ratio.

\subsubsection{Effect of underlying model assumption on $L_{500}$}

In order to explore the effect of our choice of $M_{500}-Y_{X}$ relation, we iteratively estimated $L_{500}$ by instead adopting the Malmquist bias uncorrected $L-M$ relation derived from the $M_{500}-Y_{\mathrm{X}}$ relation with self-similar slope, as given in Eq. (3) of Arnaud et al. (2010). We find that for $296(91)$ per cent of the clusters the difference is less than 5(2) per cent, with the largest differences found for low luminosity objects. Considering only clusters with $L_{500} \geq 10^{43} \mathrm{erg} \mathrm{s}^{-1}$, we find that for $\sim 99$ (95) per cent of objects the difference is less than 5 (2) per cent.

To examine the reliability of our assumption concerning the Malmquist bias correction of the $L-M$ relation, we repeated our iterative procedure by using the Malmquist bias corrected relation of Pratt et al. (2009), finding essentially the same $L_{500}$ (relative differences are $\sim 1$ per cent). This is expected, because the steep drop of the typical cluster luminosity profile with radius makes $L_{500}$ rather insensitive to the exact choice of $R_{500}$.

For the same reason we find negligible changes in the iteratively estimated $L_{500}$ if $L-M$ relations derived from samples other than that of REXCESS are adopted. We performed our computations using the $L-M$ relations reported in Reiprich \& Böhringer (2002) and Vikhlinin et al. (2009), finding that for clusters with $L_{500} \geq 10^{43} \mathrm{erg} \mathrm{s}^{-1}$ the difference is at most 3 per cent, and for lower luminosity systems it is always less than 10 per cent.

\subsubsection{Uncertainties on $L_{500}$}

There are two main sources of uncertainty on a given value of $L_{500}$. The first is due to uncertainties in the underlying model used in the homogenisation procedure; the second is due to the measurement uncertainty in the original input catalogue.

As discussed above, the choice of $L-M$ relation has a negligible effect on the final value of $L_{500}$ due to the steep drop of the luminosity profile model with radius. For the same reason, intrinsic scatter in the adopted $L-M$ relation (Pratt et al. 2009) propagates into negligible uncertainties on $L_{500}(\leq 1$ per cent). The effect of the assumed luminosity profile model is more difficult to quantify. Firstly, the deviation of the luminosity profiles in individual objects from the average is linked to cluster dynamical state (Pratt et al. 2009; Arnaud et al. 2010), and this information is not available for most of the MCXC clusters. Secondly, uncertainties due to the assumed profile propagate differently depending on the procedure adopted to compute $L_{500}$ (procedures (a) and (b) detailed above). These uncertainties are also linked to the ratio between $R_{500}$ and the aperture in which the luminosity is originally measured in a non-trivial way. However, as we argue above, the AB-model derived from the REXCESS observations is a more accurate description of the luminosity profile of $\mathrm{X}$-ray clusters than the $\beta$-model that is conventionally used.

Most importantly, the measurement uncertainties in the original input catalogues were estimated in a number of very different ways. Some input catalogues assume simple counting errors; some take into account assumptions on the source extent; some have no uncertainties at all. On average the measurement uncertainties given in the input catalogues range from around 15 to 20 per cent, but can vary strongly from object to object.

For all of these reasons, we conclude that the $L_{500}$ uncertainties are impossible to evaluate in the self-consistent way required for a homogeneous meta-catalogue, and thus the MCXC does not include this information.

\subsection{Total masses}

Total masses $M_{500}$, estimated for the same cosmology adopted here, are directly available only for MACS_MJFV and MACS_BRIGHT clusters. For almost all the clusters we therefore rely on luminosity as a mass proxy and estimate $M_{500}$ (M_500 in Table 2) using Eq. (2). The uncertainty on $M_{500}$ is dominated by the intrinsic dispersion in the $L-M$ relation (Pratt et al. 2009). While our computation of $L_{500}$ does not depend on the details of the adopted $L-M$ relation (see discussion above), the estimated $M_{500}$ obviously does. In particular, the $M_{500}$ values provided by the MCXC rely on the assumption that on average the Malmquist bias for the samples used to construct the MCXC is the same as that of the REXCESS sample. Since the selection functions of the samples we use are complex (and indeed, in most cases are not known or available), our mass estimates must rely on this assumption. In addition, while our choice ensures maximal selfconsistency in our modelling, other calibrations of the $L-M$ relation could be adopted. Nevertheless, given our estimated $L_{500}$, the computation of total masses from a different $L-M$ relation 
is straightforward. Finally, from the mass $M_{500}$ we estimate the characteristic radii $R_{500}$ (R_500 in Table 2) using Eq. (3).

\subsection{Notes}

We gather together useful information concerning individual objects and add it to the MCXC as notes (NOTES in Table 2). In the input catalogues this information is usually provided as notes and comments and because it is different in type and size from catalogue to catalogue its homogenisation is not straightforward. In general, we choose not to include detailed and extended information and we therefore refer the reader to the cited papers for more information, e.g., the notes to Table 1 in Gioia \& Luppino (1994) or in Table 2 of Romer et al. (2000a). In the following we describe the type of information we included in NOTES. For the meaning of abbreviations we refer the reader to the cited papers of each sub-catalogue.

For BCS, SGP, SHARC, NEP, MACS, CIZA, and EMSS no information is provided or is too detailed to be added in concisely. For NORAS we take information from column ID in Table 1 of Böhringer et al. (2000a) (information on source identification). For REFLEX, (Böhringer et al. 2004a), where the provided information is fairly detailed, we merge the following: (i) information in column $\mathrm{Cm}$ of Table 1 (information on source identification); (ii) the information concerning groupings as given in Table 10 with the simple note GR1, GR2,..., GR10 if the cluster is listed in one of the 10 groupings listed in the table; (iii) multipeak information as given in Table 11 (columns Morphology and Orientation are merged, as e.g., two maxima/NE-SW); and (iv) information on whether the cluster is part of a line of sight structure as given in Table 12 (we simply add losStr if the cluster appears in the table). For 400SD clusters we take the information given in the column Notes in Table 4 of Burenin et al. (2007, information on alternative names is not used). For 160SD clusters we take the information given in the column Notes in Table 4 of Mullis et al. (2003, information on alternative names is not used).

\subsection{Scale}

In order to facilitate the conversion between angular and physical sizes (e.g., for $R_{500}$ ) we provide the angular scale factor SCALE in kpc/arcsec (see Table 2).

\section{Duplicate clusters}

The overlap between the survey areas of the input catalogues induces duplicate (and in some cases triplicate, quadruplicate, etc.) entries in the MCXC catalogue. Here we explain how multiple entries are identified and which entry is retained in the MCXC. For a given cluster, according to criteria based on the type of data and the size of the input catalogue, we retain only one entry in the MCXC. Quantities from different input catalogues are not mixed or merged: for multiple input entries we retain all physical quantities (coordinates, redshift, luminosity, etc.) as given in the selected input catalogue. The full list of 2397 clusters without removal of multiple entries can be requested from the authors.

The most important criterion that we use to decide which duplicate cluster is preserved in the MCXC is the size of the input catalogue. In addition we give higher priority to catalogues that provide aperture luminosities because they ensure the most reliable and self-consistent computation of $L_{500}$. These two criteria allow us to rank the input catalogues from highest to lowest priority as in Table 1. Obviously, because of its size and the type of information it provides, NORAS/REFLEX is the catalogue with the highest priority. It is followed by other large and welldefined catalogues such as the 400SD, 160SD, BCS, etc. Hence, when a cluster is listed in more than one catalogue its MCXC entry corresponds to that from the higher priority catalogue. This catalogue ranking is not crucial for CIZA because the overlap of its survey area with other surveys is minimal.

Our procedure therefore reduces to the identification of multiple entries. This identification is based mainly on centroid coordinate differences, and to a lesser extent on redshift differences. Given the large number of entries, cluster identification is performed in three steps in order to progressively reduce the number of candidate multiple entries.

1. In a first step if two clusters in different catalogues have centroid offsets of less than $1^{\prime}$ and their relative redshift difference is less than 10 per cent they are identified as being the same cluster. Although this step removes a large number of duplicate entries, we compare their names and alternative names to make sure that we do not remove single entries. In the case of a doubtful association we do not remove any entry.

2. In a second step the resulting catalogue is inspected once more by carefully identifying clusters with centroid offsets of less than 5,10 , or even $20^{\prime}$, and by varying the relative redshift difference. This time consuming procedure is needed because different source analysis techniques can yield rather different centroid positions, in particular for nearby clusters. Redshift differences can be very large and we use them only as indicators and not as stringent constraints. Each multiple entry candidate is checked, with names and alternative names used to facilitate the procedure. Again in this step we are rather conservative and do not remove any cluster if the identification is not certain.

3. In a third step the cleaned catalogue is inspected once more with large allowances for centroid offsets, and any overlaps are checked by visually inspecting RASS and PSPC maps. The associations inspected in this last step are either multiple systems or entries where very different redshifts are given for the same X-ray cluster.

In each of the three steps we make some exceptions to the general rules explained above. First, when the redshift difference is large we keep the cluster with more reliable redshift measurement. This explains why, although it has the highest priority, the NORAS/REFLEX catalogue finally contains 11 clusters less than before our handling of duplicates. We choose the most reliable redshift measurement by taking into account the information given in the input catalogues and related papers (notes in Tables, discussion of individual objects, redshift references, etc.). If no specific note is available, we assume that the most recent measurement is the most reliable. A second exception to the general selection criteria concerns double or multiple systems, for which we retain measurements for each of the components instead of measurements of the whole system. If possible, we compared our duplicates identifications with those in other work (e.g., Mullis et al. 2003) and find perfect agreement.

A total of $558 \mathrm{MCXC}$ entries list the properties of a cluster that is a member of more than one input catalogue, and for which the information from only one input catalogue has been retained. For these entries there are therefore $N$ measurements of each of the cluster quantities (one provided by the MCXC and $N-1$ unused overlaps). There are 5 clusters with $N=5,8$ with $N=4$, 59 with $N=3$, and 486 with $N=2$. 
We find that the distribution of the ratio between the redshift given in the discarded input catalogue entry and that given in the MCXC has a median equal to 1 and is very narrow (the robust standard deviation computed using the IDL routine ROBUST_SIGMA is 0.2 per cent $^{8}$ ), but there are some large outliers. In Appendix B we discuss these outliers individually. We focus on the 25 duplicates with redshifts differing by more than an extreme factor of 1.1, and illustrate in detail how the input catalogue providing the most reliable redshift measurement was selected.

We find that positional differences between duplicates are always less than $0.2(0.5) \times R_{500}$ in 91 (99) per cent of the cases, i.e. they typically lie well within a cluster core radius. Duplicates are further discussed in Sect. 5.3, where they are used to investigate the robustness of the $L_{500}$ estimates.

In order to retain useful information, when an entry with no available alternative name is kept in the MCXC catalogue while the one we discard provides it, we copy this information into the retained entry.

The MCXC provides information concerning multiple entries in the input catalogues though the label CAT_OV (see Table 2) which contains the name of the sub-catalogue from which the removed cluster entry is a member.

\section{Discussion}

\subsection{Global catalogue characteristics}

The final MCXC is constructed from the input catalogues discussed in Sect. 2 with information homogenised as explained in Sect. 3. Multiple entries in the resulting catalogue are handled as described in Sect. 4. This procedure yields the final MCXC catalogue, which comprises in total 1743 clusters (2397 initially, see Table 1) and contains virtually no multiple entries. In the following we illustrate some basic properties of the MCXC. Because of the priorities we assign to the input catalogues, NORAS/REFLEX clusters constitute a large fraction of the MCXC (see Table 1).

The MCXC redshift histogram is illustrated in the bottom right hand panel of Fig. 1: 282, 77, and 18 clusters $(\sim 16,4,1$ per cent, respectively) have redshifts higher than $0.3,0.5$ and 0.7. In Fig. 3 we show the number of clusters as a function of luminosity: 846, 64 ( $\sim 49,4$ per cent, respectively) of the clusters have $0.1-2.4 \mathrm{keV}$ band luminosities $L_{500}$ larger than $1,10 \times 10^{44} \mathrm{erg} \mathrm{s}^{-1}$. In Fig. 4 we show the $0.1-2.4 \mathrm{keV}$ band luminosities $L_{500}$ of the $1743 \mathrm{MCXC}$ clusters as a function of redshift in log-log (top panel) and the more conventional linlog scale (bottom panel). These figures highlight both the different nature of RASS-based and serendipitous surveys and their complementarity. For a given redshift, serendipitously discovered clusters are less luminous than those from RASS-based catalogues because the deeper exposures allow lower flux limits to be adopted. This implies that the fraction of high redshift clusters in serendipitous surveys is much higher than that of RASS-based surveys. In addition to redshift and luminosity (and total mass), a fundamental quantity provided by the MCXC is the cluster position in the sky, both in equatorial and galactic coordinates. In Fig. 5 we show the distribution on the sky of the 1743 MCXC clusters in galactic coordinates. Some distinctive features are: NORAS/REFLEX, BCS and MACS clusters are fairly homogeneously distributed; the only clusters at low galactic latitude

\footnotetext{
8 This is unsurprising since the source of the redshift measurement is frequently the same.
}

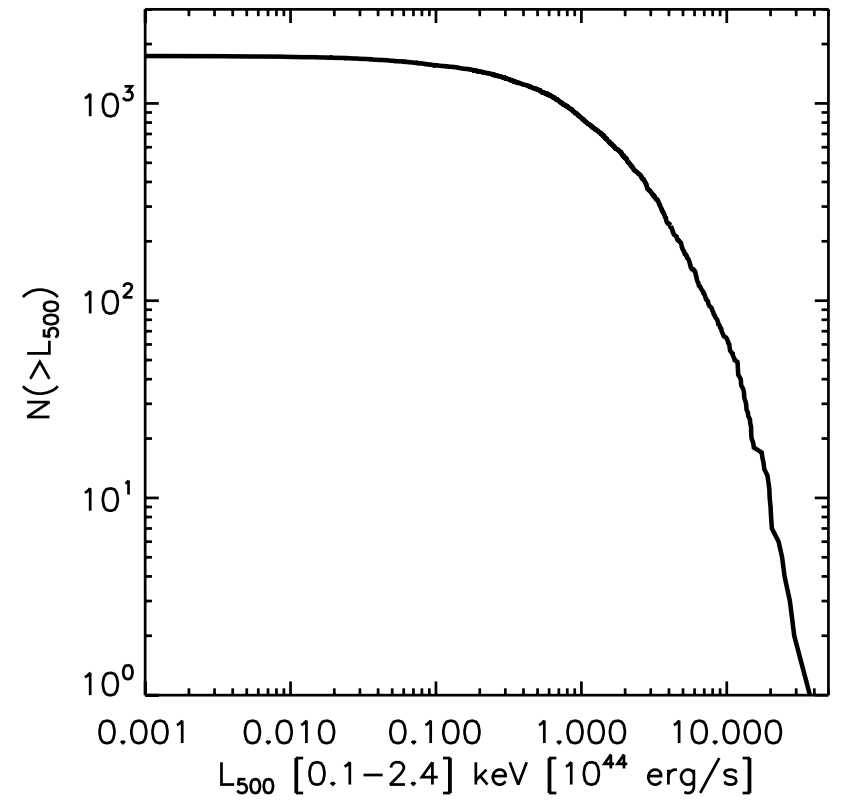

Fig. 3. Luminosity distribution of MCXC clusters.

are from the CIZA survey; the RASS-based clusters of SGP and NEP are localised in narrow regions; serendipitous clusters are sparsely distributed across the sky.

\subsection{Robustness of luminosity measurements}

As the luminosity $L_{500}$ the most relevant physical quantity provided by the MCXC, we focus on its discussion in the remainder of this section.

Since the modelling adopted in Sect. 3 is based on results from the REXCESS sample, and that the latter is a subsample of REFLEX, the comparison of the $L_{500}$ values derived in this work and those given in Pratt et al. (2009) for the 31 REXCESS clusters provides a useful test for our procedure. We remind the reader that for all the REFLEX clusters we computed the luminosities $L_{500}$ from aperture luminosities by means of the iterative procedure explained in Sect. 3. We find that our derived $R_{500}$ is larger than $R_{\text {ap }}$ for only seven REXCESS clusters, and at most only by $\sim 20$ per cent. In Fig. 6 we show the ratio between our estimate of $L_{500}$ and the XMM-Newton measurements given in Pratt et al. (2009), L500,REXCESS. Uncertainties on the luminosity ratios are computed from quadratic sum of the errors given in Pratt et al. (2009) and propagation of the aperture luminosity errors provided in Böhringer et al. (2004a). For one cluster the redshift adopted in our work differs from the one used in

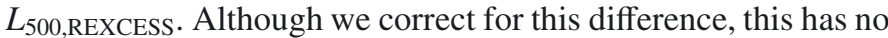
impact on our results. Symbols in Fig. 6 are as in Pratt et al. (2009), i.e. blue stars for cool core clusters and red squares for morphologically disturbed clusters. We compute error weighted means and standard deviations of the luminosity ratio and find: $0.965 \pm 0.141$ for all 31 clusters, $0.932 \pm 0.078$ for the cool core clusters, and $0.951 \pm 0.193$ for the disturbed clusters. Our comparison indicates a good agreement between the two measurements. Notice the fairly large scatter at low luminosity, the large scatter for disturbed clusters with respect to cool core clusters, and that there is an indication that our luminosity estimates are on average biased low in cool core systems (a $1 \sigma$ effect). The 
R. Piffaretti et al.: The MCXC
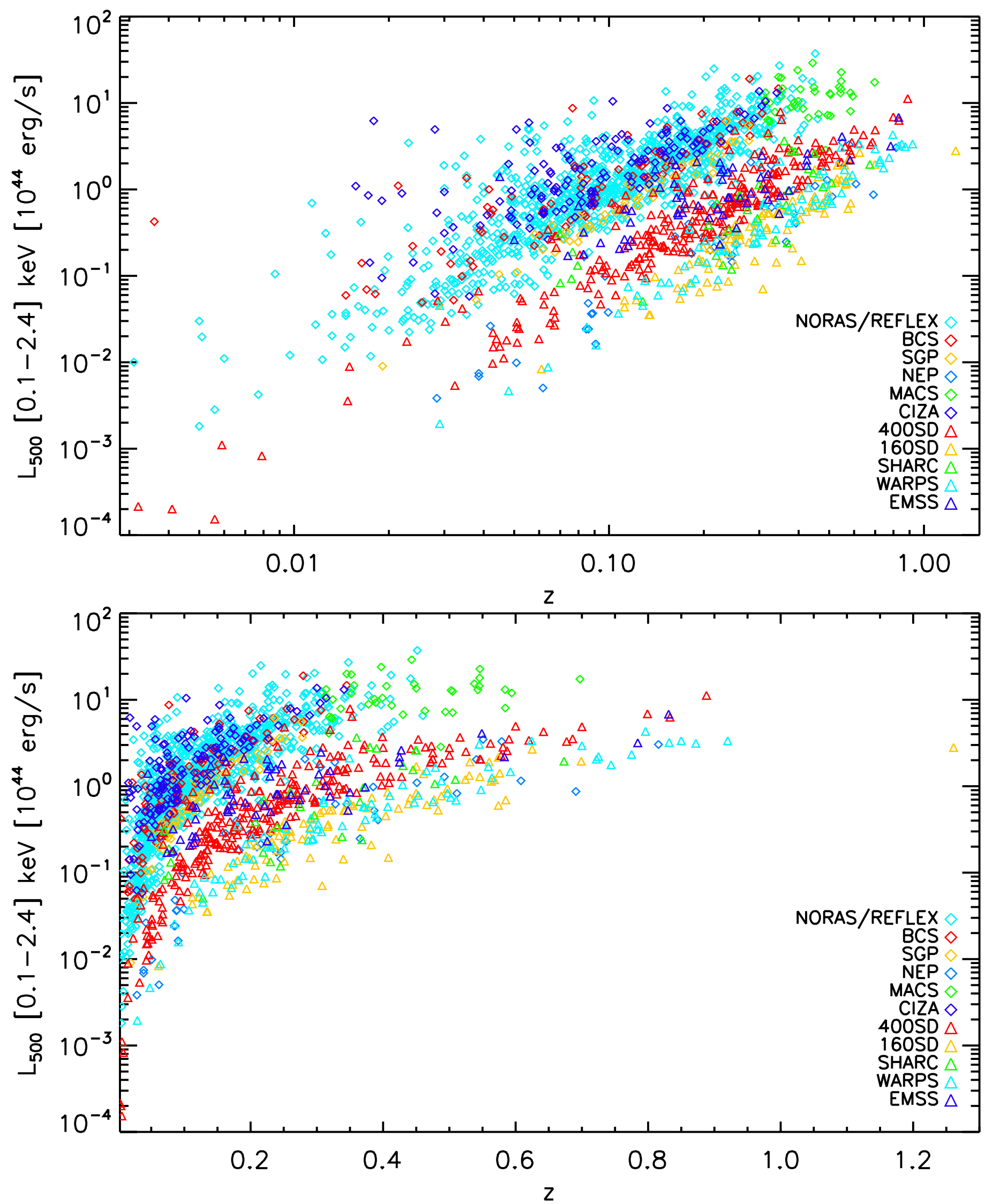

Fig. 4. Top: the $0.1-2.4 \mathrm{keV}$ band luminosities $L_{500}$ of the $1743 \mathrm{MCXC}$ clusters as a function of redshift. Diamonds and triangles indicate clusters from RASS-based and serendipitous catalogues, respectively. Bottom: same, but in lin-log scale. 


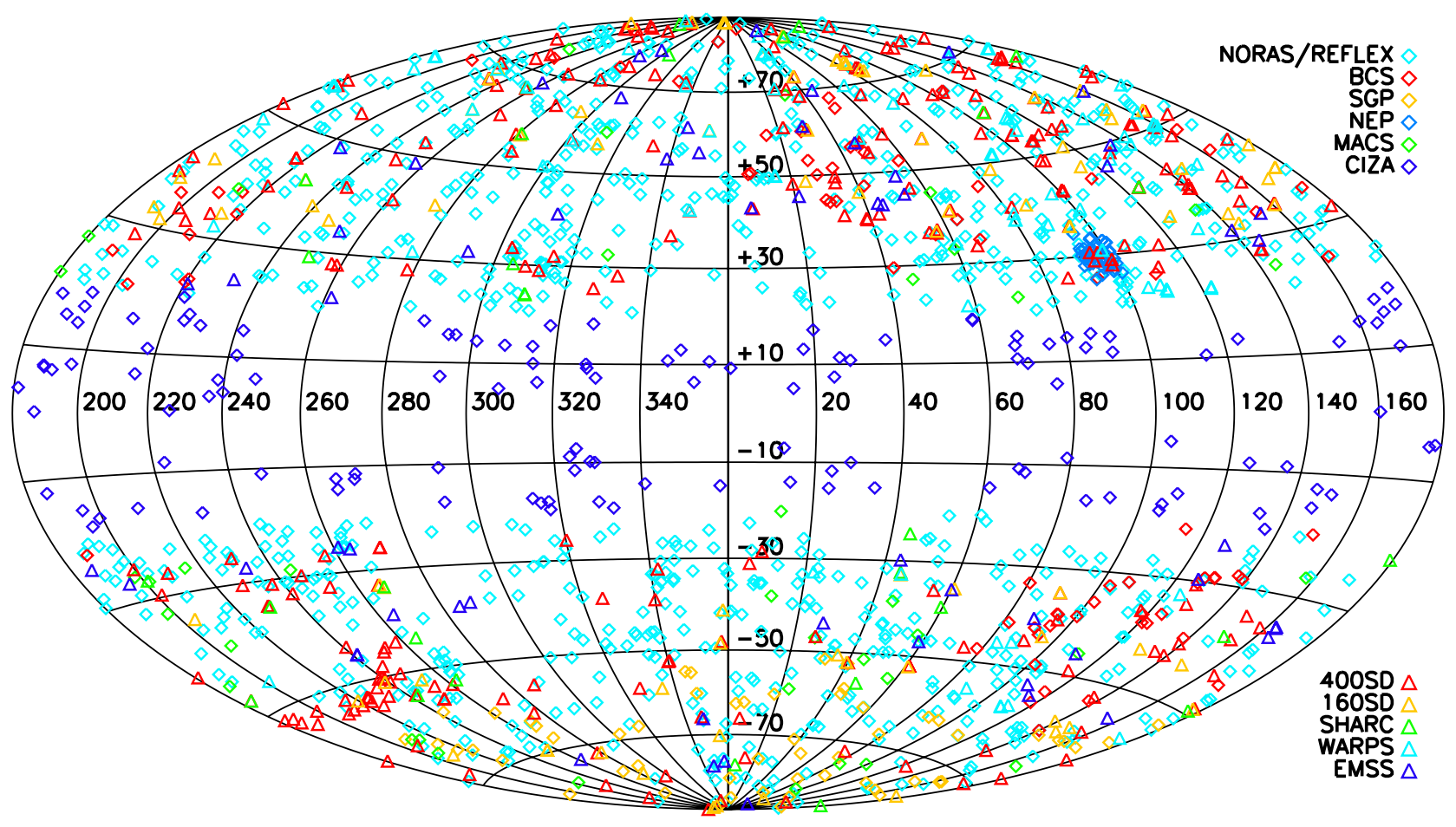

Fig. 5. Sky map of the 1743 MCXC clusters in galactic coordinates. Symbols and colors are the same as in Fig. 4.

lower luminosity ratio for cool core clusters is expected because they are modeled using the AB-model derived from the mean of the REXCESS sample (Eq. (1)), although their emission is more centrally peaked. We find no trend of luminosity ratio with the ratio $R_{\text {ap }} / R_{500}$ and no redshift dependence as the REXCESS sample redshift leverage is too small.

\subsection{Intercomparison of original luminosity measurements}

The procedure adopted to handle multiple entries (detailed in Sect. 4 ) allows us to compare $L_{500}$ estimates derived from different input luminosity measurements. For each of the $558 \mathrm{MCXC}$ clusters that are member of more than one input catalogue we compute the ratio $L_{500} / L_{500, \text { MCXC }}$ where $L_{500, \mathrm{MCXC}}$ is the luminosity given in the MCXC and $L_{500}$ is the luminosity of the same cluster, but derived from a different input catalogue (i.e., the overlap luminosity). As explained in Sect. 4, in some cases the redshifts provided by the input catalogues can be fairly different. We therefore correct the luminosities of the overlaps by multiplying them with the squared ratio of the luminosity distances at the two different redshifts. This is equivalent to comparing the $0.1-2.4 \mathrm{keV}$ band fluxes within $R_{500}$. The MCXC provides these luminosity ratios though the quantity L_500_RAT (see Table 2) where they are ordered in the same way as the sub-catalogue names in CAT_OV.

In Fig. 7 we show the luminosity $L_{500}$ of the overlaps (top panel) and the ratio $L_{500} / L_{500, \mathrm{MCXC}}$ (bottom panel, in dex units) as a function of $L_{500, \mathrm{MCXC}}$. Both a direct fit to the data (which is shown in the top panel of the figure and basically indistinguishable from equality) and the mean value of the luminosity ratios indicate that the different luminosity determinations are in excellent agreement. The error weighted mean and standard deviation of the luminosity ratio $L_{500} / L_{500, \text { MCXC }}$ are adopted to quantify the agreement between the different luminosity estimates. Errors are computed from the uncertainties quoted in the input catalogues, assuming that the relative error on the luminosities $L_{500}$ is the same as that of the input luminosities. We find 1.03 and 0.27 for the error weighted mean and standard deviation, respectively.

In Fig. 8 we show the ratio $L_{500} / L_{500, \mathrm{MCXC}}$ as a function of $z$, the cluster redshift given in the MCXC. A visual inspection of the Figure indicates that there is no significant trend between the two quantities. We performed a linear fit to the data shown in the bottom panel of Fig. 7 and in Fig. 8 and, in both cases, we find a slope consistent with zero, i.e. find no statistically significant trend of the luminosity ratio with either luminosity or redshift. We performed the same analysis by taking into account whether the compared luminosities are derived from RASS or pointed observations, and whether they are computed iteratively or just by adopting a constant conversion factor (see Sect. 3), and find no significant trend.

The luminosity comparison therefore shows that on average the agreement between different $L_{500}$ measurements is excellent. However, the clear outliers in Fig. 7 indicate that for some clusters there are large discrepancies.

Although a discussion on individual objects, and thus on the difference between specific survey measurements, is beyond the scope of our work, we briefly discuss very discrepant luminosity estimates by focussing on strong outliers with luminosity ratios larger than 2 or smaller 0.5 in Fig. 7 (i.e., differences larger than a factor of 2$)$. There is a total of twenty objects ( $\sim 4$ per cent 
of those with more than one luminosity measurement) of such discrepant estimates. For six of these clusters, three luminosity estimates are available. Interestingly, of these, we always find that only one of the three is very different from the others, and that the two remaining estimates agree within a few per cent. For the other fourteen clusters only two estimates of $L_{500}$ are available. Of these, five involve measurements from the EMSS and seven are faint objects at low redshift, where extrapolation might strongly affect the luminosity estimates. For the remaining two clusters (A2507 and RXC J1003.0+3254) we find no obvious explanation.

\section{Summary and conclusions}

Motivated by the strong need for a large, homogeneous compilation in the framework of X-ray, SZ and other multiwavelength studies, we have presented the construction and properties of the MCXC, a Meta-Catalogue of X-ray detected Clusters of galaxies. The MCXC is constructed from publicly-available RASS-based (NORAS, REFLEX, BCS, SGP, NEP, MACS, and CIZA) and serendipitous (160SD, 400SD, SHARC, WARPS, and EMSS) cluster catalogues (see Sect. 2). The information from these input catalogues is systematically homogenised using the most up to date knowledge of the structural properties and scaling relations of X-ray clusters, and is undertaken in a self-consistent way (see Sect. 3). More specifically, in addition to the fairly straightforward standardisation of quantities such as coordinates and redshifts (RAJ2000, DEJ2000, etc., and Z in Table 2, we converted the available luminosities to $0.1-2.4 \mathrm{keV}$ band luminosities $L_{500}$ ( L_500 in Table 2 ) by adopting the average gas density profile (Croston et al. 2008) and $L-M$ relation (Pratt et al. 2009) derived from the representative X-ray cluster sample REXCESS. The computation is performed directly from aperture luminosities when available ( $\sim 6$ per cent of the MCXC clusters) and we verify that the derived luminosities do not depend on the details of the adopted $L-M$ relation.

Total masses $M_{500}$ and radii $R_{500}$ can be computed from the luminosities $L_{500}$ by adopting an $L-M$ relation. The MCXC provides these quantities computed self-consistently using the $L-M$ relation adopted in this work (M_500 and R_500 in Table 2). The MCXC further provides three cluster identifiers: the MCXC name, the original name as given in the input catalogues, and an alternative name (NAME_MCXC, NAME, and NAME_ALT in Table 2, respectively). The latter has been homogenised to match both SIMBAD and NED standards. In addition, we collated important information usually provided as notes and comments in the input catalogues (NOTES in Table 2).

Multiple entries originating from overlaps between the survey areas of the input catalogues are very carefully handled (see Sect. 4). The result of this procedure is provided by the MCXC (CAT_OV in Table 2). We compare luminosity measurements from different catalogues, finding that on average the agreement is excellent, and discuss the most discrepant measurements (see Sect. 5). We find good agreement with the precisely measured REXCESS luminosities given in Pratt et al. (2009). These comparisons strongly support the validity of our approach. The MCXC provides the luminosity ratios for clusters that appear in multiple input catalogues (see L_500_RAT in Table 2).

The MCXC comprises 1743 clusters ordered by right ascension, and contains virtually no multiple entries. The full MCXC is available at $\mathrm{CDS}^{9}$ and contains the information given in Cols. (1)-(19) in Table 2, where the first 40 entries are given as an example.

\footnotetext{
9 http://cds.u-strasbg.fr/
}

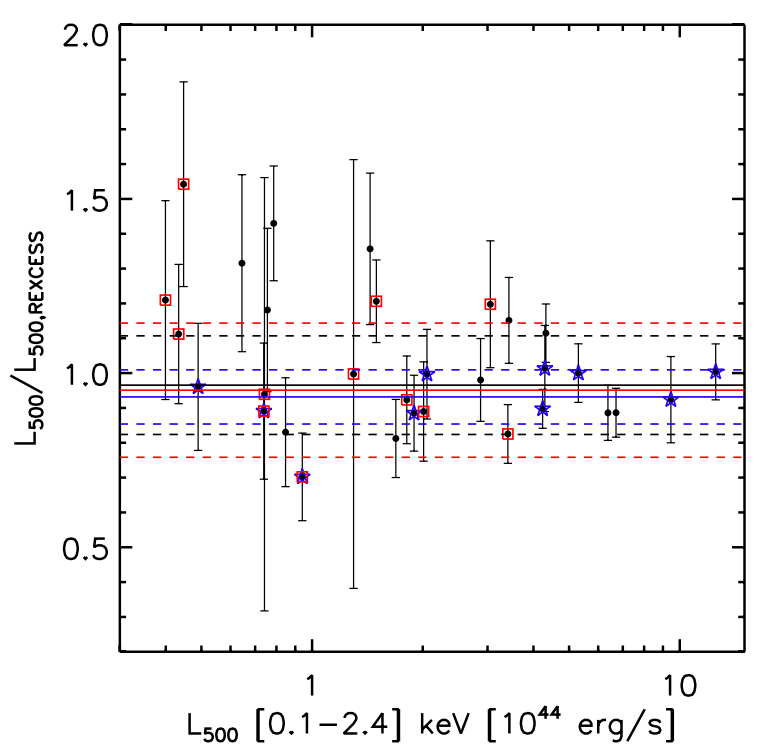

Fig. 6. Ratio $L_{500} / L_{500, \text { REXCESS }}$ between our estimate of the $0.1-2.4 \mathrm{keV}$ band luminosities $L_{500}$ and the XMM-Newton measurements of Pratt et al. (2009) as a function of $L_{500}$. Blue stars indicate cool core clusters while red squares morphologically disturbed clusters. Solid lines indicate error weighted means and dashed lines represent the error weighted means \pm error weighted standard deviations (black for all clusters, blue for cool core clusters, and red for morphologically disturbed clusters as defined in Pratt et al. 2009).

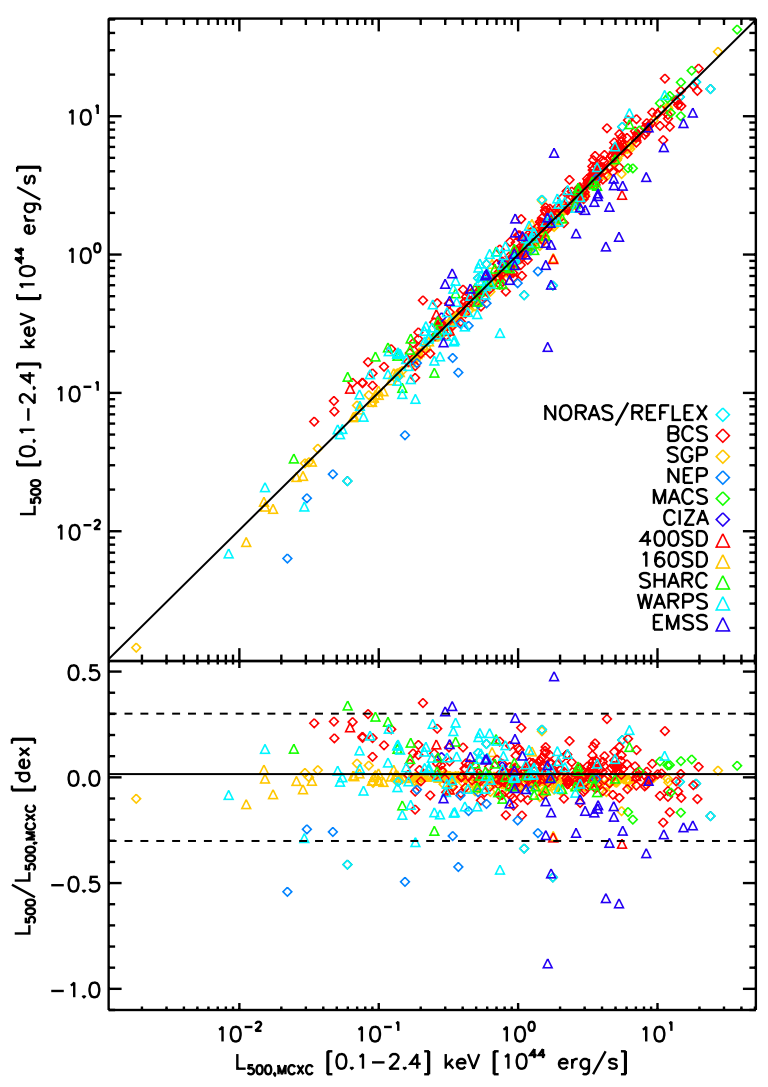

Fig. 7. Luminosity $L_{500}$ of the overlaps (top panel) and the ratio $L_{500} / L_{500, \text { MCXC }}$ in dex (bottom panel) as a function of $L_{500, \text { MCXC }}$, the luminosity measurements retained in the MCXC. Symbols and colors are the same as in Fig. 4, but refer to the overlaps only. The solid line in the top panel indicates the best fit to the data. The solid line in the bottom panel indicates the error weighted mean of the luminosity ratio, while the dashed horizontal lines indicates the luminosity ratios equal to 2 and 0.5 . 


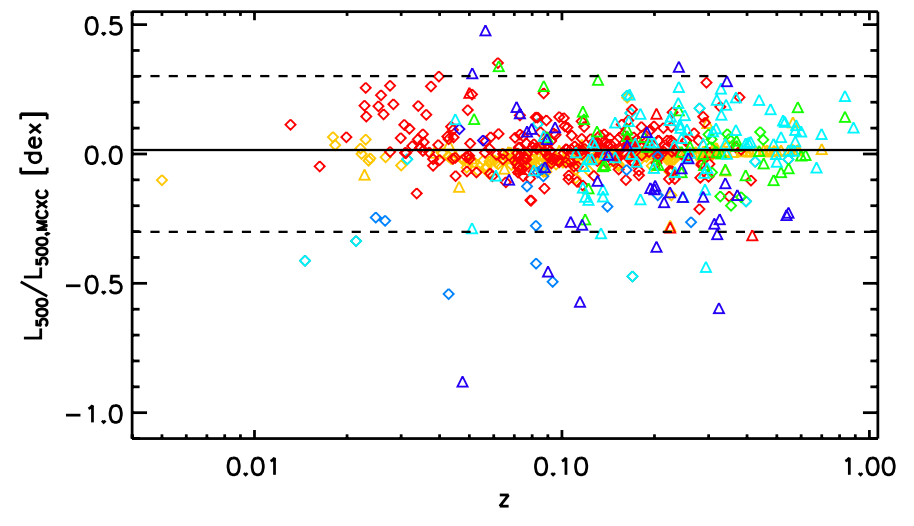

Fig. 8. Ratio $L_{500} / L_{500, \mathrm{MCXC}}$ in dex as a function of redshift. Symbols, colors, and lines are the same as in Fig. 7.

The work by Melin et al. (2011) is one example of how the information provided by the MCXC can be used for SZ studies. In particular, their work illustrates that given the MCXC luminosities, the universal pressure profile and the associated SZ scaling relations provided by Arnaud et al. (2010) can be used to yield the X-ray predicted SZ signal from individual objects, which can be then compared to that observed with WMAP. A further, more extensive, example of the MCXC in use is provided by the recent papers released by the Planck Collaboration (Planck Collaboration 2011c,d,e,f,g,h). In particular, in Planck Collaboration (2011d), Planck SZ candidate sources are crosscorrelated with the MCXC to find known X-ray clusters. In Planck Collaboration (2011g), the MCXC is cross-correlated with the XMM-Newton archive to yield a list of observed X-ray clusters detected at high signal to noise in the Planck survey. Finally, in Planck Collaboration (2011f), the Planck SZ signal at the position of all objects in the MCXC is bin-averaged in X-ray luminosity and compared to X-ray model predictions.

The MCXC is an ongoing project and will be extended to include available data for individual clusters at high redshift (the most relevant for cosmological studies), and cluster catalogues derived from ongoing X-ray surveys, when they are publicly available.

Acknowledgements. We thank Isabella Gioia, Patrick Henry, and Donald Horner for helpful discussions and Nabila Aghanim for strongly supporting the project. This research has made use of the VizieR database, operated at CDS, Strasbourg. E.P. acknowledges the support of grant ANR-06-JCJC-0141.

\section{Appendix A: AB-model versus $\beta$-model luminosity profiles}

In the following we illustrate the difference between predictions based on the AB-model and a "typical" $\beta$-model. In particular we focus on $L_{500}$ and total luminosities $L_{\text {tot }}$ estimated from a given aperture luminosity.

The AB-model adopted in this work is given by Eq. (1) with $x=r / R_{500}, x_{\mathrm{c}}=0.303, \alpha=0.525$, and $\beta=0.768$ (see Sect. 3 ) and we investigate $\beta$-models given by Eq. (1) with $\alpha=0, \beta=$ $2 / 3$, and $x_{\mathrm{c}}=0.05,0.1,0.2,0.3$, and $0.4\left(x_{\mathrm{c}}=r_{\mathrm{c}} / R_{500}\right.$, where $r_{\mathrm{c}}$ is the usual $\beta$-model core radius).

For all models we compute luminosity profiles (spherically symmetric) which are then cylindrically integrated to obtain "projected" luminosities as a function of cluster-centric distance. Finally these are normalised at $R_{\text {ap }}$ where $L\left(<R_{\text {ap }}\right)=L_{\text {ap }}$ and shown in Fig. A. 1 for $R_{\mathrm{ap}}=0.5 \times R_{500}$. The Figure shows that, with respect to the AB-model, $\beta$-models with small core radii

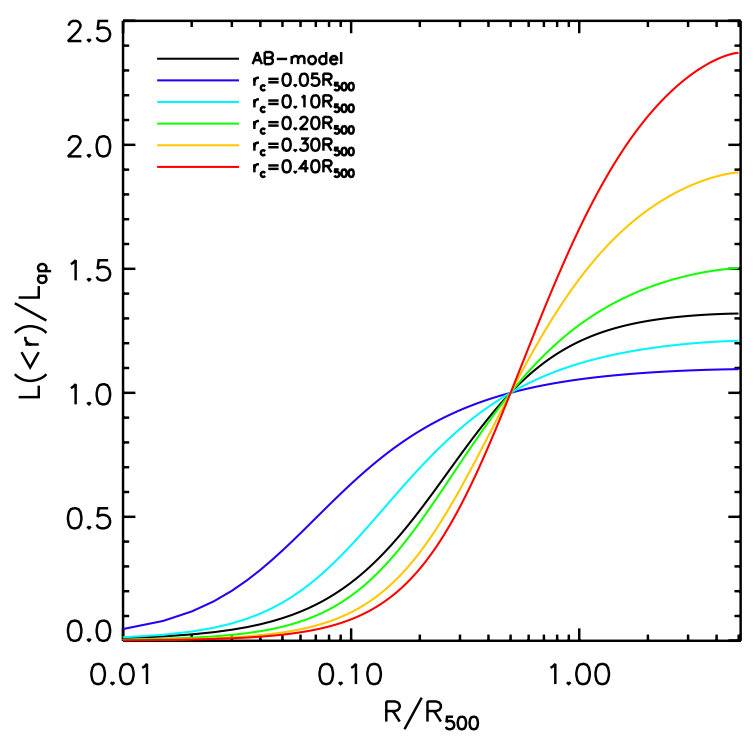

Fig. A.1. Luminosity radial profile normalised at $R_{\text {ap }}=0.5 \times R_{500}$ for the AB-model (black line) and $\beta$-models with different core radii (color lines).

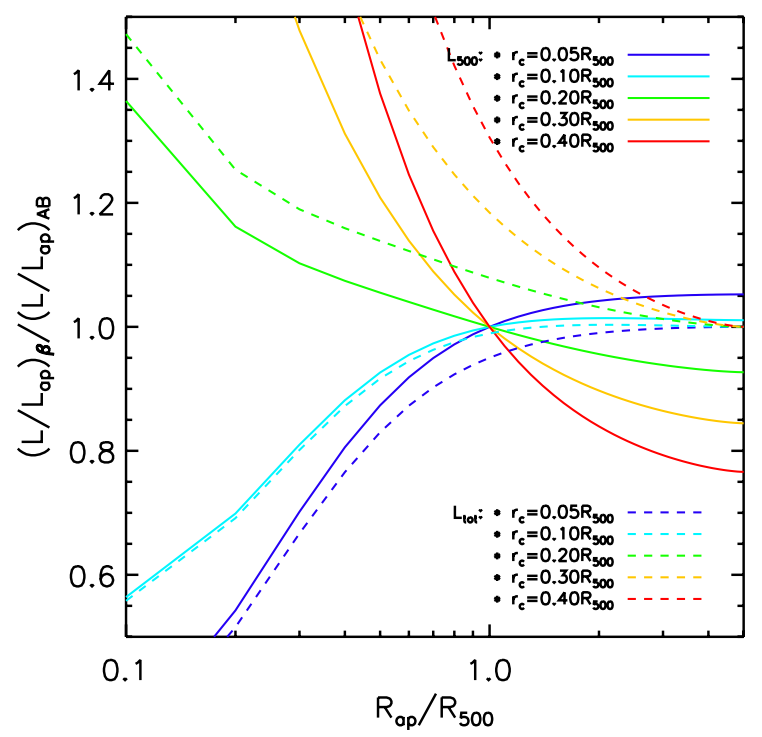

Fig. A.2. The $\beta$-model to $\mathrm{AB}$-model ratio of the normalised luminosity profile $L / L_{\text {ap }}$ evaluated at $R_{500}$ (solid lines) and $5 \times R_{500}$ (dashed lines) as a function of $R_{\mathrm{ap}}$.

yield centrally concentrated luminosity profiles, which in turn are shallow at large radii. The opposite is true for $\beta$-models with large core radii, which predict very extended profiles. With respect to the AB-model, a $\beta$-model with $x_{\mathrm{c}}=0.05$ underestimates $L_{500}$ by $\sim 20$ per cent while for $x_{\mathrm{c}}=0.4$ it yields a factor of 2 larger value.

We investigate the effect of modelling on global luminosities by computing $L_{500}$ and $L_{\mathrm{tot}}=L\left(<5 \times R_{500}\right)$ as a function of $R_{\text {ap }}$. In Fig. A. 2 we show the $\beta$-model to AB-model ratio of $L / L_{\text {ap }}$ (the normalised luminosity profile) evaluated at $R_{500}$ (i.e. $L=L_{500}$, solid lines) and $5 \times R_{500}$ (i.e. $L=L_{\mathrm{tot}}$, dashed lines) as a function of $R_{\text {ap }}$. With respect to the AB-model, $\beta$-models with small/large core radii underestimate/overestimate $L_{500}$ for apertures smaller than $R_{500}$, while for $R_{\text {ap }}>R_{500}$ this behavior is reversed. Total luminosities $L_{\text {tot }}$ are always higher/lower than the AB-model estimates for $\beta$-models with small/large core radii and the difference increases with decreasing $R_{\mathrm{ap}}$. 
R. Piffaretti et al.: The MCXC

Table B.1. Duplicate clusters with redshift measurements differing by more than 10 per cent. Column labels as for Table 2.

\begin{tabular}{|c|c|c|c|c|c|c|c|c|c|}
\hline & \multicolumn{5}{|c|}{ MCXC } & \multicolumn{4}{|c|}{ OVERLAP } \\
\hline & NAME & SUB_CATALOGUE & RAJ2000 & DEJ2000 & $\bar{Z}$ & NAME & SUB_CATALOGUE & $\bar{Z}$ & $\begin{array}{c}\text { separation } \\
\text { (arcmin) }\end{array}$ \\
\hline 1 & RX J0210.2-3932 & 160SD & 21013.8 & -393251 & 0.1680 & WARP J0210.2-3932 & WARPS & 0.1900 & 0.02 \\
\hline 2 & RX J0210.4-3929 & 160SD & 21025.6 & -392947 & 0.1650 & WARP J0210.4-3929 & WARPS & 0.2730 & 0.40 \\
\hline 3 & BVH2007 126 & 400SD_SER & 112745.4 & 43947 & 0.3440 & MS1125.3+4324 & EMSS_1994 & 0.1810 & 3.53 \\
\hline 4 & BVH2007 132 & 400SD_SER & 114216.6 & 10272 & 0.1170 & RX J1142.2+1027 & SHARC_BRIGHT & 0.0698 & 0.26 \\
\hline 5 & BVH2007 132 & 400SD_SER & 114216.6 & 10272 & 0.1170 & WARP J1142.2+1026 & WARPSII & 0.1500 & 0.11 \\
\hline 6 & BVH2007 184 & 400SD_SER & 141013.4 & 594238 & 0.3160 & RX J1410.2+5942 & 160SD & 0.2500 & 0.13 \\
\hline 7 & BVH2007 185 & 400SD_SER & 141015.8 & 593827 & 0.3190 & RX J1410.2+5938 & 160SD & 0.2500 & 0.11 \\
\hline 8 & BVH2007 198 & 400SD_SER & 152440.3 & 95735 & 0.5160 & RX J1524.6+0957 & SHARC_BRIGHT & 0.0780 & 0.23 \\
\hline 9 & BVH2007 219 & 400SD_SER & 181910.1 & 69939 & 0.2050 & RX J1819.0+6909 & NEP & 0.0880 & 0.59 \\
\hline 10 & A1185 & BCS & 111046.8 & 284222 & 0.0314 & RXC J1110.5+2842 & NORAS & 0.0220 & 3.18 \\
\hline 11 & A1367 & BCS & 114436.5 & 194532 & 0.0214 & RXC J1144.7+1949 & NORAS & 0.0276 & 3.90 \\
\hline 12 & RXJ0142.0+2131 & eBCS & 1422.6 & 213119 & 0.2803 & RXC J0142.0+2130 & NORAS & 0.0698 & 0.67 \\
\hline 13 & RXJ1836.5+6344 & eBCS & 183630.5 & 634453 & 0.0834 & RXC J1836.5+6344 & NORAS & 0.0484 & 0.36 \\
\hline 14 & A2507 & eBCS & 225657.1 & 53249 & 0.1696 & RXC J2256.8+0530 & NORAS & 0.1960 & 2.40 \\
\hline 15 & MACSJ0358.8-2955 & MACS_BRIGHT & 35854.4 & -295532 & 0.4250 & RXCJ0358.8-2955 & REFLEX & 0.1681 & 0.87 \\
\hline 16 & MACSJ2211.7-0349 & MACS_BRIGHT & 221145.9 & -34942 & 0.3970 & RXCJ2211.7-0350 & REFLEX & 0.2700 & 0.76 \\
\hline 17 & MACSJ1149.5+2223 & MACS_MJFV & 114935.3 & 22249 & 0.5450 & RXC J1149.5+2223 & NORAS & 0.1761 & 0.56 \\
\hline 18 & RXC J1421.6+3717 & NORAS & 142141.4 & 371745 & 0.1813 & A1902 & BCS & 0.1600 & 0.32 \\
\hline 19 & RXC J1601.3+5354 & NORAS & 16123.4 & 535422 & 0.1068 & A2149 & eBCS & 0.0675 & 1.96 \\
\hline 20 & RXC J1715.2+0309 & NORAS & 171512.8 & 3947 & 0.1647 & RXJ1715.2+0309 & $\mathrm{eBCS}$ & 0.1317 & 0.19 \\
\hline 21 & RXC J2104.9+1401 & NORAS & 21454.2 & 14140 & 0.1615 & $\mathrm{ZwCl} 8484$ & eBCS & 0.2029 & 0.32 \\
\hline 22 & RXCJ0152.9-1345 & REFLEX & 15259.0 & -134512 & 0.0050 & RXC J0152.9-1345 & SGP & 0.0057 & 0.21 \\
\hline 23 & RXCJ0322.2-5310 & REFLEX & 32212.7 & -531041 & 0.0797 & MS0320.9-5322 & EMSS_1994 & 0.0710 & 0.43 \\
\hline 24 & RXCJ2248.7-4431 & REFLEX & 224843.5 & -443144 & 0.3475 & RXC J2248.7-4431 & SGP & 0.1495 & 0.06 \\
\hline 25 & RXCJ2321.4-2312 & REFLEX & 232124.3 & -231220 & 0.0890 & RXC J2321.4-2312 & SGP & 0.1870 & 0.42 \\
\hline
\end{tabular}

\section{Appendix B: Duplicate clusters with discrepant redshift measurements}

The 1743 object MCXC described in this paper is available at CDS. As described in Sect. 4, construction of the MCXC includes the identification and removal of multiple entries from the full list of clusters resulting from combination of all input catalogues. The full compilation of 2397 clusters without removal of multiple entries can be requested from the authors.

Here, we discuss in detail duplicate clusters for which the redshift given in the discarded input catalogue entry and that given in the MCXC differ by more than 10 per cent. As discussed in Sect. 4, the overall agreement in redshift between duplicates is excellent, and there are only 25 objects fulfilling this criterion in the MCXC. In Table B.1 we list cluster name, sub-catalogue, coordinates, and redshift as given in the MCXC, together with cluster name, sub-catalogue, and redshift of the discarded overlap. The last column of the Table gives the angular separation between the duplicates. We notice that BVH2007 132 is a triplicate (rows 4 and 5 in Table B.1 ). In the following we provide information on redshift estimates of these duplicate clusters individually and justify our selection of the reliable redshift measurement.

1. The WARPS redshift estimate is uncertain (see note in Table 5 in Perlman et al. 2002a).

2. The discrepancy between 160SD and WARPS redshifts is discussed in Mullis et al. (2003).

3. The 400SD redshift estimates are based on higher quality data (Abazajian et al. 2005) than those used in the EMSS (Gioia \& Luppino 1994). In addition, this object is discussed in the notes to Table 1 in Gioia \& Luppino (1994).

4. The discrepancy between 400SD and SHARC redshifts is discussed in Sect. 4.1 of Burenin et al. (2007).

5. The WARPSII redshift for this cluster is not secure (see Sect. 3.2 in Horner et al. 2008).
6. The 400SD redshift estimates are based on higher quality data (Abazajian et al. 2005) than those used in the 160SD (Huchra et al. 1990).

7. Same as for 6 (BVH2007 184).

8. The average temperature of $5.1 \mathrm{keV}$ measured by Vikhlinin et al. (2002) rules out the low redshift estimate. The redshift quoted in the 400SD is in excellent agreement with the one given in 160SD and WARPSII.

9. Redshifts in the 400SD (see Table 4 in Burenin et al. 2007) and NEP (Gioia et al. 2003) were obtained from a dedicated follow-up programmes. The 400SD is the more recent.

10. The BCS redshift reference (Huchra et al. 1992) is more recent than the NORAS one (Struble \& Rood 1991).

11. Same as for 10 (A1185).

12. Both eBCS and NORAS quote redshift measurements obtained from their optical follow-up programmes. The eBCS measurement is the most recent and has subsequently been confirmed by Barr et al. (2005).

13. Same as for 12 (RXJ0142.0+2131). In this case the eBCS redshift measurement is in excellent agreement with the one given in NEP.

14. The eBCS redshift was obtained from a dedicated follow-up programme (see Table 1 in Ebeling et al. 2000a) and is more recent than the redshift references adopted for NORAS (see Table 1 in Böhringer et al. 2000a).

15. Redshifts of MACS clusters are obtained from recent, dedicated follow-up observations (Ebeling et al. 2010).

16. Same as for 15 (MACSJ0358.8-2955).

17. Same as for 15.

18. The NORAS redshift was obtained from a dedicated followup programme (see Table 1 in Böhringer et al. 2000a) and is more recent than the redshift references adopted for BCS (see Table 3 in Ebeling et al. 1998).

19. The NORAS redshift reference (year 2000; see Table 1 in Böhringer et al. 2000a) is more recent than the one adopted for the eBCS (year 1991; see Table 1 in Ebeling et al. 2000a). 
20. The NORAS redshift was obtained from a dedicated followup programme (see Table 1 in Böhringer et al. 2000a) and is more recent than the redshift references adopted for eBCS (see Table 1 in Ebeling et al. 2000a).

21. Same as for 20 (RXC J1715.2+0309).

22. The REFLEX redshift reference (year 2002; see Table 6 in Böhringer et al. 2004a) is more recent than the one adopted for the SGP (year 1991; see Table 3 in Cruddace et al. 2002a).

23. The REFLEX redshift reference (Böhringer et al. 2004a) is more recent than the one in the EMSS (Gioia \& Luppino 1994).

24. The REFLEX redshift was obtained from a dedicated followup programme (see Table 6 in Böhringer et al. 2004a) and is more recent than the redshift references adopted for SGP (see Table 3 in Cruddace et al. 2002a).

25. The REFLEX redshift reference (year 2002; see Table 6 in Böhringer et al. 2004a) is more recent than the redshift references adopted for SGP (year 1991; see Table 3 in Cruddace et al. 2002a).

\section{References}

Abazajian, K., Adelmam McCarthy, J. K., Agüeros, M. C., et al. 2005, AJ, 129, 1755

Andersson, K., Benson, B. A., Ade, P. A. R., et al. 2011, ApJ, 738, 48 Arnaud, M., Pointecouteau, E., \& Pratt, G. W. 2007, A\&A, 474, L37 Arnaud, M., Pratt, G. W., Piffaretti, R., et al. 2009, A\&A, 517, A92 Barkhouse, W. A., Green, P. J., Vikhlinin, A., et al. 2006, ApJ, 645, 955 Barr, J., Davies, R., Jørgensen, I., Bergmann, M., \& Crampton, D. 2005, AJ, 130, 445

Böhringer, H., Briel, U. G., Schwarz, R. A., et al. 1994, Nature, 368, 828 Böhringer, H., Voges, W., Huchra, J. P., et al. 2000a, ApJS, 129, 435

Böhringer, H., Voges, W., Huchra, J. P., et al. 2000b, VizieR Online Data Catalog, 212, 90435

Böhringer, H., Schuecker, P., Guzzo, L., et al. 2004a, A\&A, 425, 367

Böhringer, H., Schuecker, P., Guzzo, L., et al. 2004b, VizieR Online Data Catalog, 342, 50367

Böhringer, H., Schuecker, P., Pratt, G. W., et al. 2007, A\&A, 469, 363

Burenin, R. A., Vikhlinin, A., Hornstrup, A., et al. 2007, ApJS, 172, 561

Burenin, R. A., Vikhlinin, A., Hornstrup, A., et al. 2009, VizieR Online Data Catalog, 217, 20561

Burke, D. J., Collins, C. A., Sharples, R. M., Romer, A. K., \& Nichol, R. C. 2003a, MNRAS, 341, 1093

Burke, D. J., Collins, C. A., Sharples, R. M., Romer, A. K., \& Nichol, R. C. 2003b, VizieR Online Data Catalog, 734, 11093

Carlstrom, J. E., Ade, P. A. R., Aird, K. A., et al. 2011, PASP, 123, 568

Croston, J. H., Pratt, G. W., Böhringer, H., et al. 2008, A\&A, 487, 431

Cruddace, R., Voges, W., Böhringer, H., et al. 2002a, ApJS, 140, 239

Cruddace, R., Voges, W., Böhringer, H., et al. 2002b, VizieR Online Data Catalog, 214, 239

Cruddace, R., Voges, W., Böhringer, H., et al. 2003, ApJS, 144, 299

Ebeling, H., Edge, A. C., Bohringer, H., et al. 1998, MNRAS, 301, 881

Ebeling, H., Edge, A. C., Allen, S. W., et al. 2000a, MNRAS, 318, 333

Ebeling, H., Edge, A. C., Boehringer, H., et al. 2000b, VizieR Online Data Catalog, 730, 10881

Ebeling, H., Edge, A. C., Allen, S. W., et al. 2000c, VizieR Online Data Catalog, 731,80333

Ebeling, H., Edge, A. C., \& Henry, J. P. 2001, ApJ, 553, 668

Ebeling, H., Mullis, C. R., \& Tully, R. B. 2002, ApJ, 580, 774

Ebeling, H., Barrett, E., Donovan, D., et al. 2007, ApJ, 661, L33

Ebeling, H., Edge, A. C., Mantz, A., et al. 2010, MNRAS, 407, 83

Evrard, A. E., Metzler, C. A., \& Navarro, J. F. 1996, ApJ, 469, 494

Fassbender, R., 2007, Ph.D. Thesis [astro-ph/0806.0861v1]

Fowler, J. W., Niemack, M. D., Dicker, S. R., et al. 2007, Appl. Opt., 46, 3444

Gioia, I. M., Maccacaro, T., Schild, R. E., et al. 1990, ApJS, 72, 567

Gioia, I. M., \& Luppino, G. A. 1994, ApJS, 94, 583

Gioia, I. M., Henry, J. P., Mullis, C. R., et al. 2003, ApJS, 149, 29

Henry, J. P. 2000, ApJ, 534, 565

Henry, J. P. 2004, ApJ, 609, 603

Henry, J. P., Mullis, C. R., Voges, W., et al. 2006, ApJS, 162, 304
Horner, D. J., Perlman, E. S., Ebeling, H., et al. 2008, ApJS, 176, 374

Horner, D. J., Perlman, E. S., Ebeling, H., et al. 2009, VizieR Online Data Catalog, 217, 60374

Huchra, J. P., Henry, J. P., Postman, M., \& Geller, M., J. 1990, ApJ, 365, 66

Huchra, J. P., Geller, M. J., Clemens, C. M., Tokarz, S. P., \& Michel, A. 1992, Bulletin d'Information du Centre de Donnees Stellaires, 41, 31

Kocevski, D. D., Ebeling, H., Mullis, C. R., \& Tully, R. B. 2007, ApJ, 662, 224

Komatsu, E., Smith, K. M., Dunkley, J., et al. 2010, ApJSS, 192, 18

Kravtsov, A. V., Vikhlinin, A., \& Nagai, D. 2006, ApJ, 650, 128

Liedahl, D. A., Osterheld, A. L., \& Goldstein, W. H. 1995, ApJ, 438, L115

Mahdavi, A., Hoekstra, H., Babul, A., \& Henry, J. P. 2008, MNRAS, 384, 1567

Majumdar, S., \& Mohr, J. J. 2003, ApJ, 585, 603

Mantz, A., Allen, S. W., Rapetti, D., \& Ebeling, H. 2010a, MNRAS, 406, 1759

Mantz, A., Allen, S. W., Ebeling, H., Rapetti, D., \& Drlica-Wagner, A. 2010b, MNRAS, 406, 1773

Markevitch, M. , \& Vikhlinin, A. 2007, Phys. Rep., 443, 1

Maughan, B. J. 2007, ApJ, 668, 772

Maughan, B. J., Jones, C., Forman, W., \& Van Speybroeck, L. 2008, ApJS, 174, 117

McNamara, B. R., \& Nulsen, P. E. J. 2007, ARA\&A, 45, 117

Melin, J.-B., Bartlett, J. G., \& Delabrouille, J. 2006, A\&A, 459, 341

Melin, J.-B., Bartlett, J. G., Delabrouille, J., et al. 2010, A\&A, 525, A139

Menanteau, F., Gonzalez, J., Juin, J.-B., et al. 2010, ApJ, 723, 1523

Meneghetti, M., Rasia, E., Merten, J., et al. 2010, A\&A, 514, A93

Mewe, R., Gronenschild, E. H. B. M., \& van den Oord, G. H. J. 1985, A\&AS, 62, 197

Mullis, C. R., McNamara, B. R., Quintana, H., et al. 2003, ApJ, 594, 154

Nagai, D., Vikhlinin, A., \& Kravtsov, A. V. 2007, ApJ, 655, 98

Pacaud, F., Pierre, M., Refregier, A., et al. 2007, MNRAS, 382, 1289

Perlman, E. S., Horner, D. J., Jones, L. R., et al. 2002a, ApJS, 140, 265

Perlman, E. S., Horner, D. J., Jones, L. R., et al. 2002b, VizieR Online Data Catalog, 214, 265

Piffaretti, R., Jetzer, P., Kaastra, J. S., \& Tamura, T. 2005, A\&A, 433, 101

Piffaretti, R., \& Valdarnini, R. 2008, A\&A, 491, 71

Planck Collaboration 2011c, A\&A, 536, A7 DOI: $10.1051 / 0004-6361 / 201116474$

Planck Collaboration 2011d, A\&A, 536, A8 DOI: 10.1051/0004-6361/201116459

Planck Collaboration 2011e, A\&A, 536, A9 DOI: 10.1051/0004-6361/201116460

Planck Collaboration 2011f, A\&A, 536, A10 DOI: $10.1051 / 0004-6361 / 201116457$

Planck Collaboration 2011g, A\&A, 536, A11 DOI: $10.1051 / 0004-6361 / 201116458$

Planck Collaboration 2011h, A\&A, 536, A12 DOI: 10.1051/0004-6361/201116489

Pointecouteau, E., Arnaud, M., \& Pratt, G. W. 2005, A\&A, 435, 1

Popesso, P., Böhringer, H., Brinkmann, J., Voges, W., \& York, D. G. 2004, A\&A 423, 449

Pratt, G. W., \& Arnaud, M. 2002, A\&A, 394, 375

Pratt, G. W., Böhringer, H., Croston, J. H., et al. 2007, A\&A, 461, 71

Pratt, G. W., Croston, J. H., Arnaud, M., \& Bohringer, H. 2009, A\&A, 498, 361

Pratt, G. W., Arnaud, M., Piffaretti, R., et al. 2010, A\&A, 511, A85

Rasia, E., Ettori, S., Moscardini, L., et al. 2006, MNRAS, 369, 2013

Rasmussen, J., \& Ponman, T.J. 2007, MNRAS, 380, 1554

Reiprich, T. H., \& Böhringer, H. 2002, ApJ, 567, 716

Romer, A. K., Nichol, R. C., Holden, B. P., et al. 2000a, ApJS, 126, 209

Romer, A. K., Nichol, R. C., Holden, B. P., et al. 2000b, VizieR Online Data Catalog, 212, 60209

Romer, A. K., Viana, P. T. P., Liddle, A. R., \& Mann, R. G. 2001, ApJ, 547, 594

Rosati, P., della Ceca, R., Norman, C., \& Giacconi, R. 1998, ApJ, 492, L21

Rosati, P., Borgani, S., \& Norman, C. 2002, ARA\&A, 40, 539

Struble, M. F., \& Rood, H. J. 1991, ApJS, 77, 363

Šuhada, R., Song, J., Böhringer, H., et al. 2010, A\&A, 514, L3

Sun, M., Sehgal, N., Voit, G. M., et al. 2011, ApJ, 727, L49

Sunyaev, R. A., \& Zel'dovich, Y. B. 1972, Comments on Astrophysics and Space Physics, 4, 173

Tauber, J. A., Mandolesi, N., Puget, J.-L., et al. 2010, 520, A1

Vanderlinde, K., Crawford, T. M., de Haan, T., et al. 2010, ApJ, 772, 1180

Vikhlinin, A., van Speybroeck, L., Markevitch, M., Forman, W. R., \& Grego, L. 2002, ApJ, 578, L107

Vikhlinin, A., Kravtsov, A., \& Forman, W. R. 2006, ApJ, 640, 691

Vikhlinin, A., Burenin, R. A., Ebeling, H., et al. 2009, ApJ, 692, 1033

Voges, W., Aschenbach, B., Boller, T., et al. 1999, A\&A, 349, 389

Voit, G. M. 2005, Rev. Mod. Phy., 77, 207

Zhang, Y.-Y., Okabe, N., Finoguenov, A., et al. 2010, ApJ, 711, 1033 